\title{
Presentation of Causal Model for Rumination Based on Negative and Positive Psychological Variables
}

\section{Arezo Shahhoseini, Davood Manavipour*}

Department of Psychology, Faculty of Psychology and Educational Sciences, Garmsar Branch, Islamic Azad University, Garmsar, Iran

\section{ABSTRACT}

Introduction: In recent years, the thought patterns in emotional disorders and its role in the continuance of these disorders has greatly drawn attention of the clinical professionals and researchers. The purpose of this study was to offer a causal model for rumination based on positive and negative psychological variables. Materials and Methods: The statistical population in this study included all the students of the Islamic Azad University of Garmsar in the academic year of 2014-2015. Out of these students, 300 persons were selected through an accessible sampling method. First, the instrument of the research was Response Styles Scale of Nolen-Hoeksema, self-compassion scale of Neff, mindfulness scale of Brown \& Ryan, and test of Self-Conscious Affect-2 of Tangney and et al. The structural components of the variables were determined using factor analysis and a conceptual model was developed based on psychological variables, then hypothetical model tested by path analysis. Results: Mindfulness directly had a negative effect on rumination and could predict it positively via brooding. Self-compassion directly had a negative effect on rumination and could predict it negatively via brooding. Shame directly had a positive effect on rumination and could predict it positively via brooding. Guilt directly had a positive effect on rumination and could predict it positively via reflection. Conclusion: Among different variables and subfactors, brooding subfactor directly and self-compassion indirectly had the highest share in predicting rumination.

\section{Key words:}

1 Regression (Psychology)

2. analysis

3. Psychology

*Corresponding Author: Davood Manavipour

E-mail: Manavipor53@yahoo.com 


\section{ارائَٔ مدل علّى براى نشخوار فكرى بر اساس متغيرهاى روانشناختى مثبت و منفى}

آرزو شاه حسينى، داود معنوى يور"

كروه روانشناسى، دانشكده روانشناسى و علوم تربيتى، واحد گرمسار، دانشكاه آزاد اسلامى، كرمسار، ايران

كليد وازهها: 1. بر ترسيون (روانشناسى)

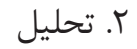

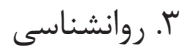

مقدمه: در سالهاى اخير الكوهاى تفكر در اختلالات هيجانى و نقش آن در تدوام اين اختلالات تا

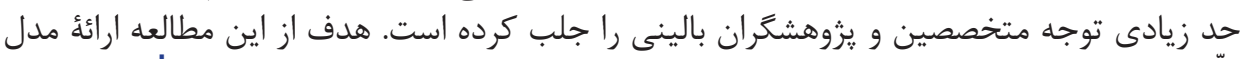

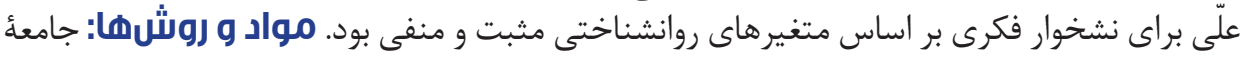

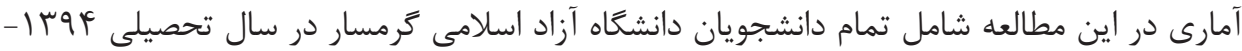

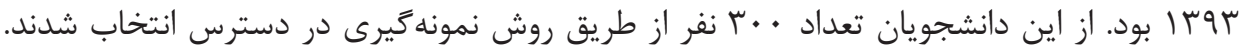

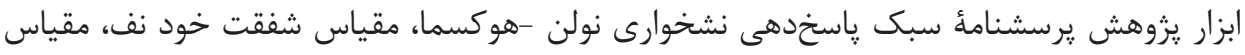

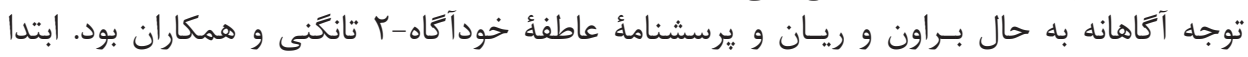

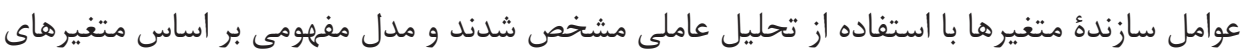

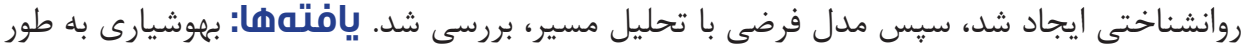

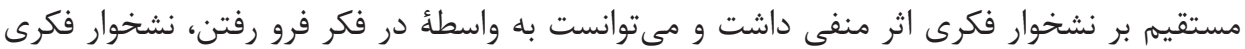

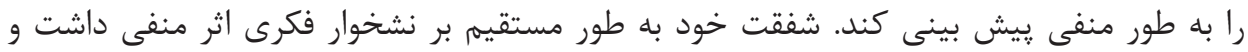

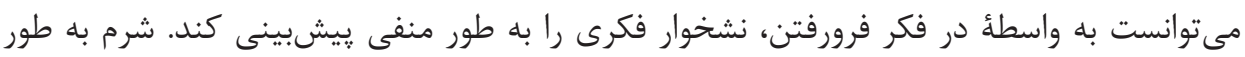

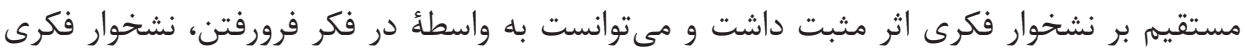

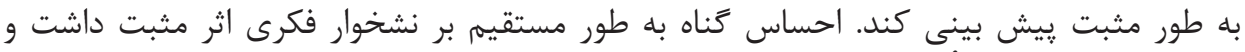

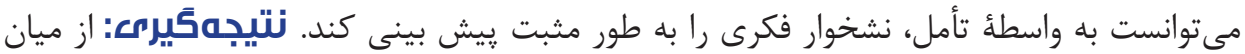

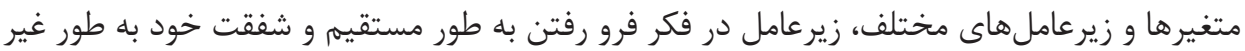

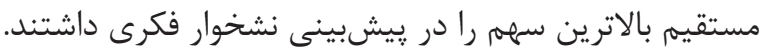

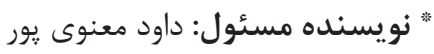
آدرس الكترونيكى: Manavipor53@yahoo.com 


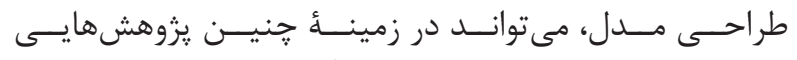

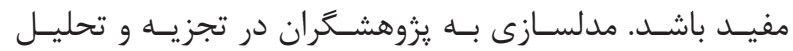

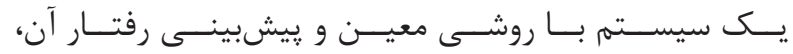

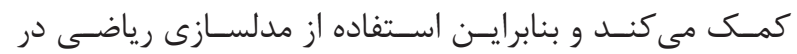

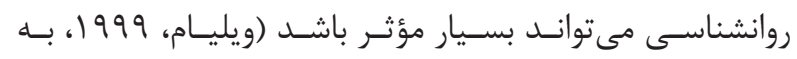

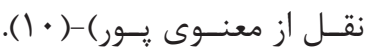

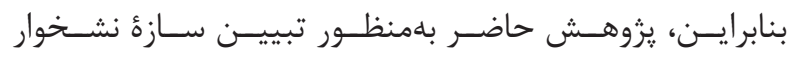

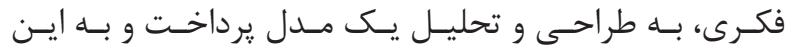

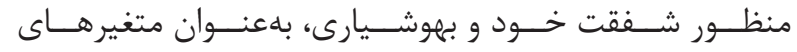

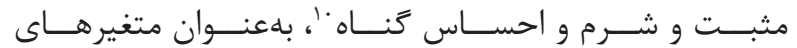

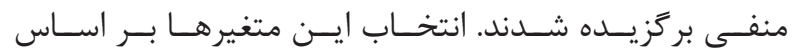

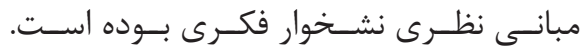

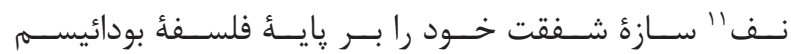

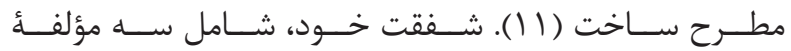

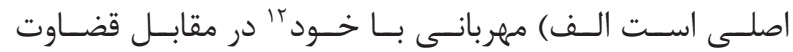

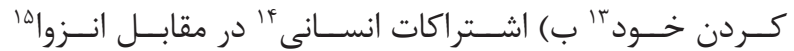

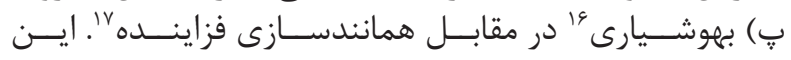

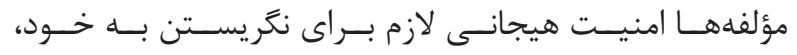

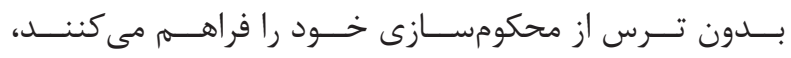

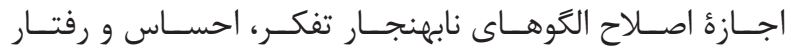

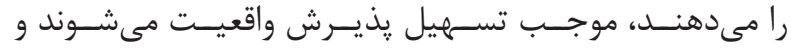

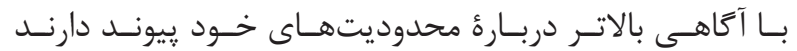

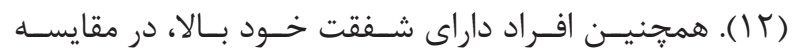

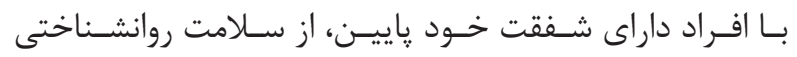

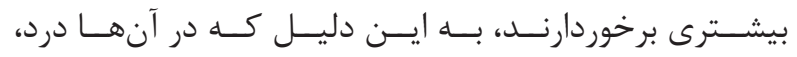

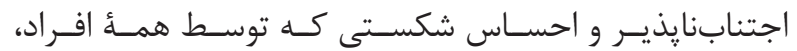

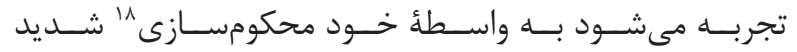

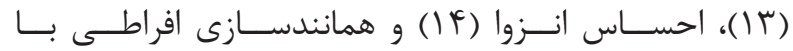

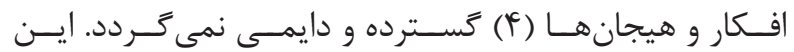

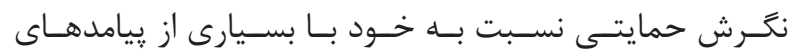

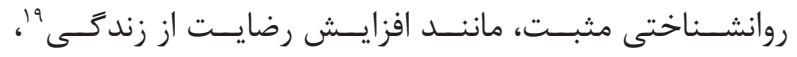

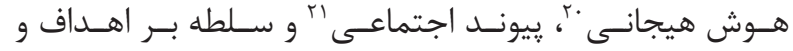

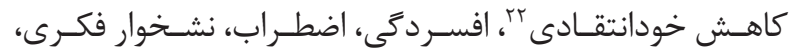
كمال

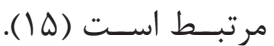

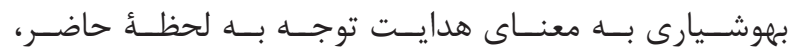

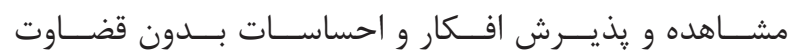

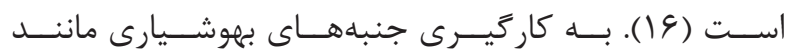

\section{${ }^{1}$ Rumination}

${ }^{2}$ Response styles theory

${ }^{3}$ Nolen-Hoksema

${ }^{4}$ Self-regulatory executive function

${ }^{5}$ Goal progress theory

${ }^{6}$ Smith and Alloy

${ }^{7}$ Self-compassion

${ }^{8}$ Mindfulness

${ }^{9}$ Shame

${ }^{10}$ Quilt

${ }^{11}$ Neff

${ }^{12}$ Self-kindness

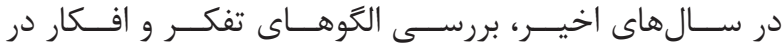

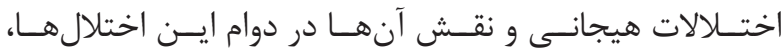

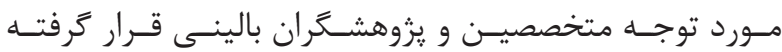

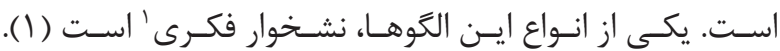

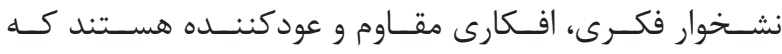

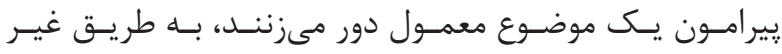

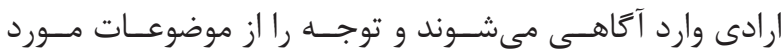

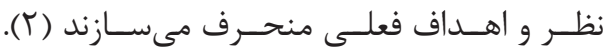

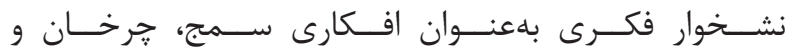

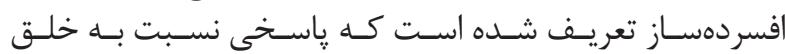

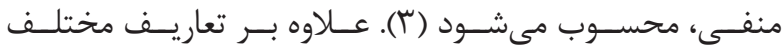

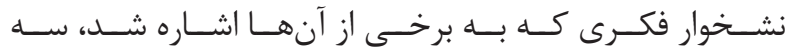

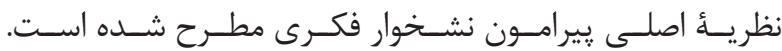

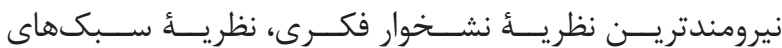

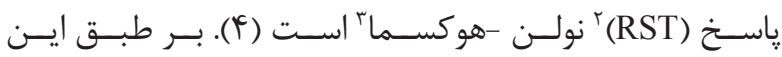

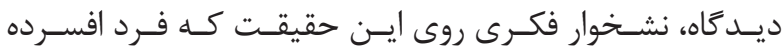

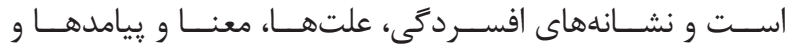

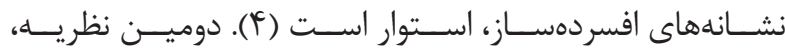

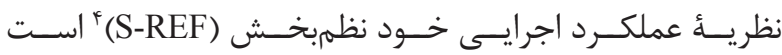

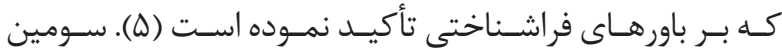

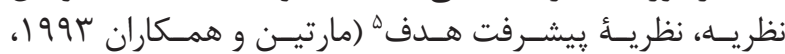

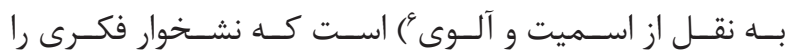

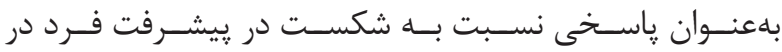

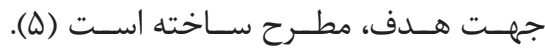

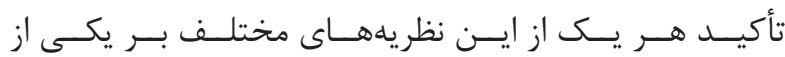

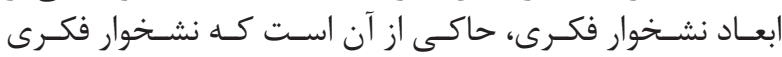

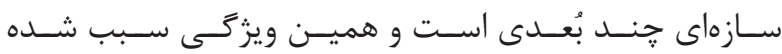

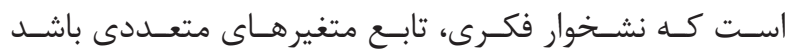

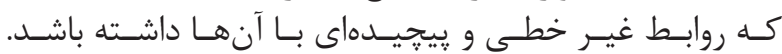

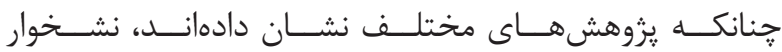

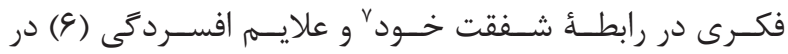

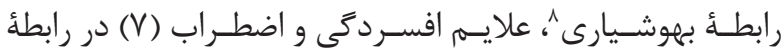

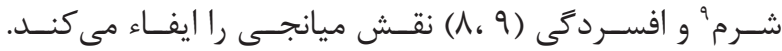

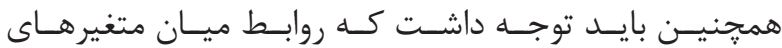

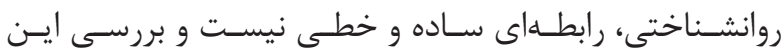

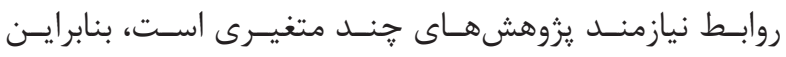

${ }^{13}$ Self-judgment

${ }^{14}$ Common humanity

${ }^{15}$ Isolation

${ }^{16}$ Mindfulness

${ }^{17}$ Over-identification

${ }^{18}$ Self-condemnation

${ }^{19}$ Life satisfaction

${ }^{20}$ Emotional intelligence

${ }^{21}$ Social connectedness

${ }^{22}$ Self-criticism

${ }^{23}$ Neurotic perfectionism

${ }^{24}$ Disordered eating behaviors 


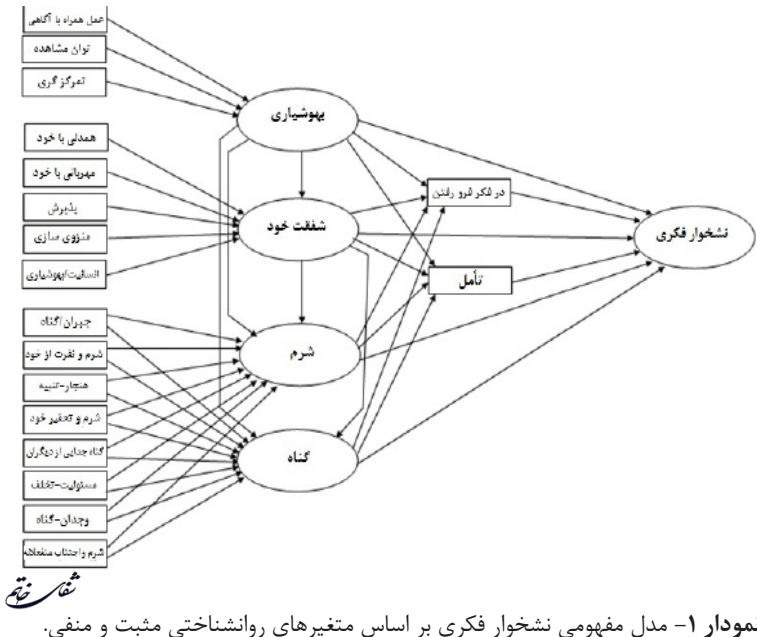

مواد و روشها روش :يزوهش، جامعلُ آمارى و نمونه

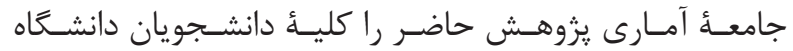

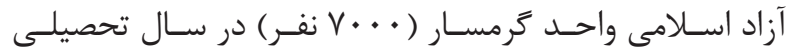
تو

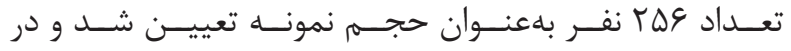

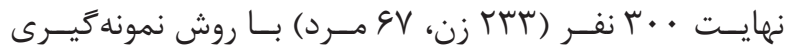

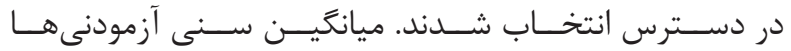

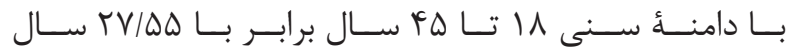

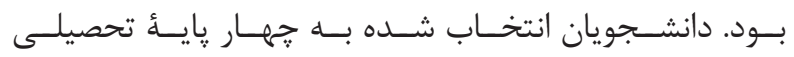

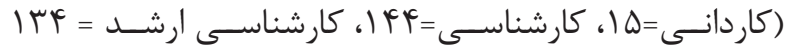

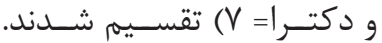

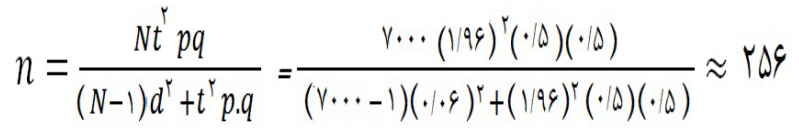

$$
\text { ابزار تر دآورى دادهها }
$$

\section{يرسشنامهُ سبك پاسخدهى نشخوارى}

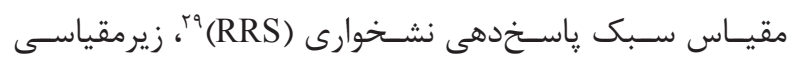

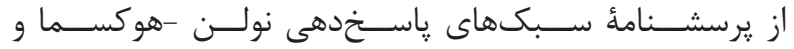

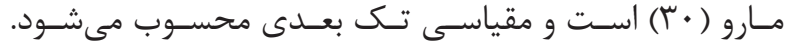

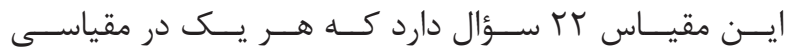

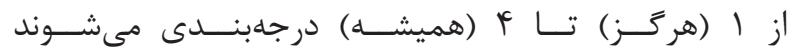

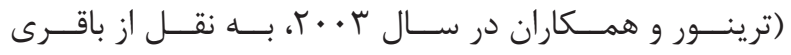

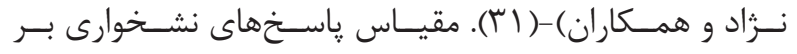

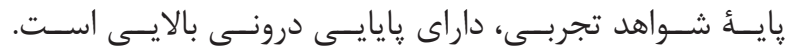

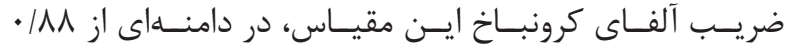

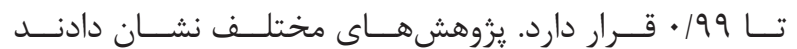

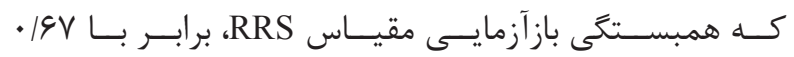

${ }^{25}$ Self- reflection

${ }^{26}$ Acceptance

${ }^{27}$ Openness

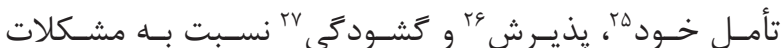

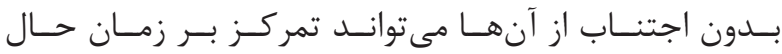

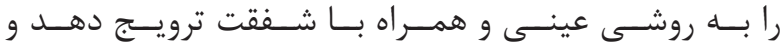

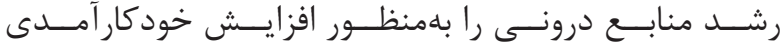

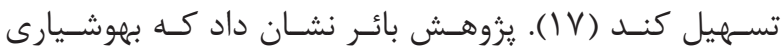

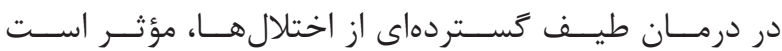

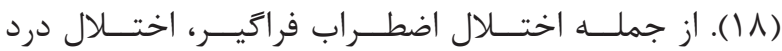

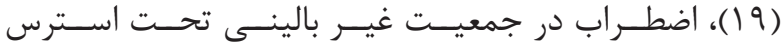

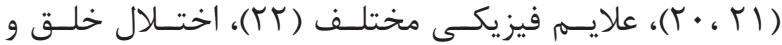

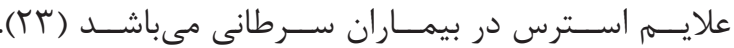

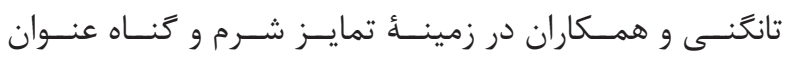

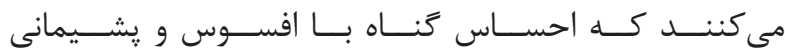

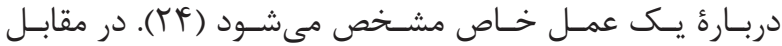

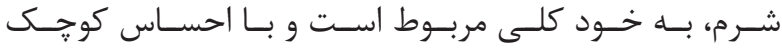

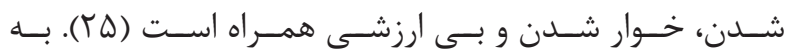

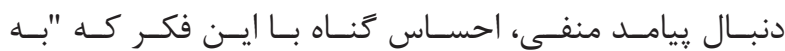

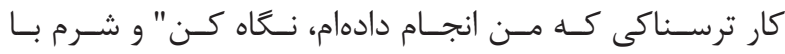

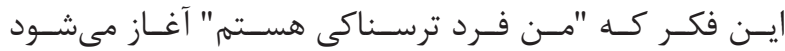

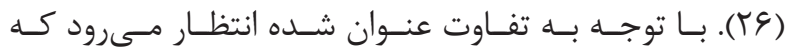

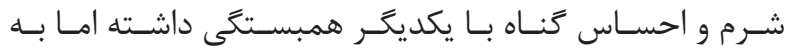

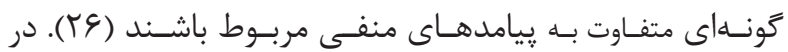

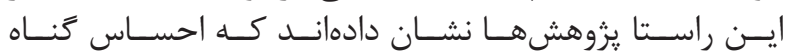

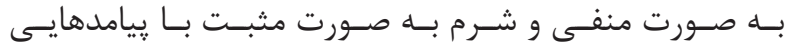

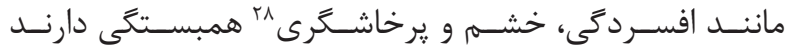

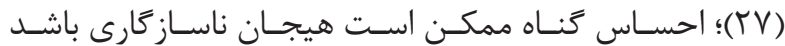

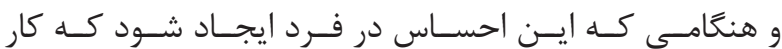

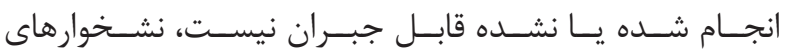

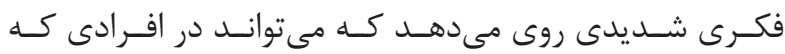

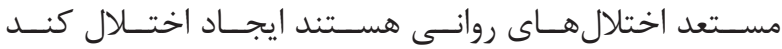

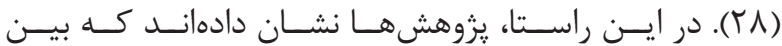

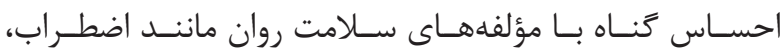

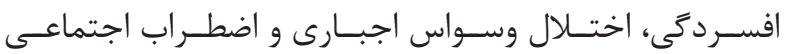

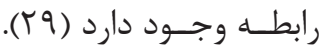

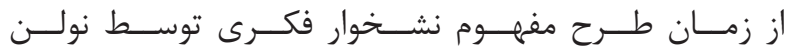

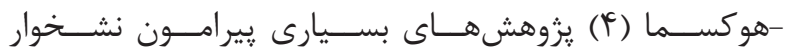

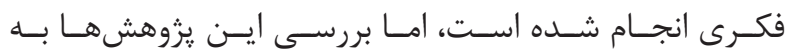

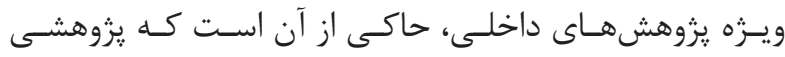

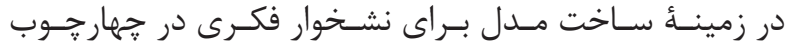

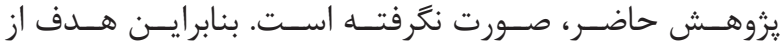

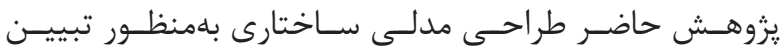

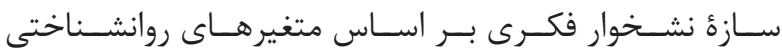

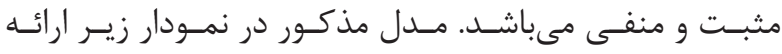

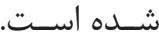

\footnotetext{
${ }^{28}$ Aggression

${ }^{29}$ Rumination response style
} 


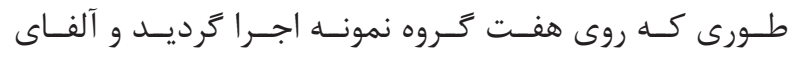

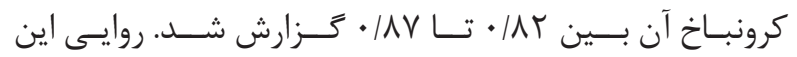

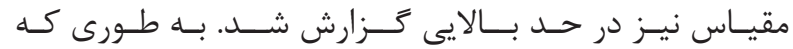

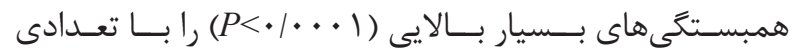

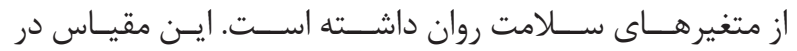

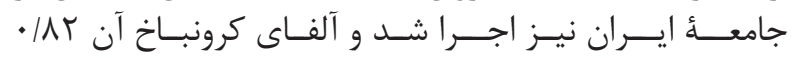

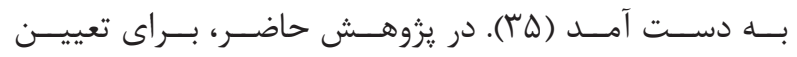

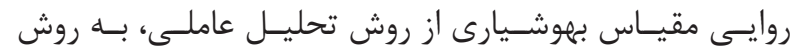

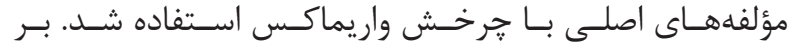

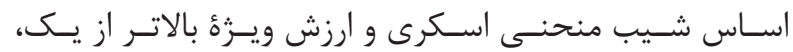

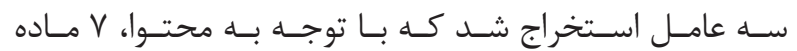

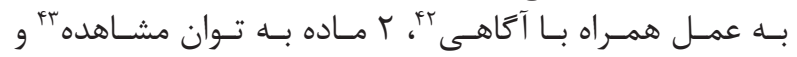

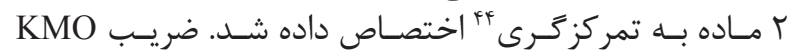

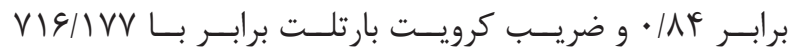

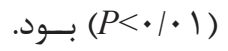

\section{r- برسشنامة عاطفة خود آكاه}

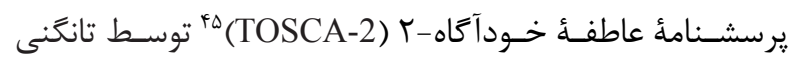

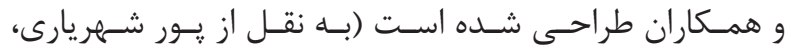

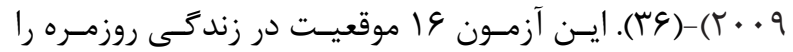

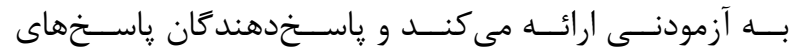

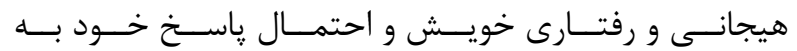

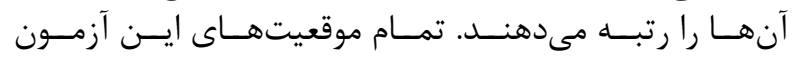

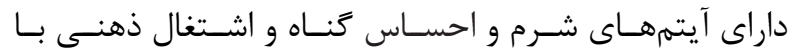

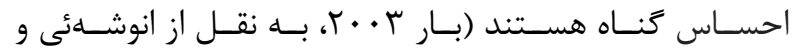

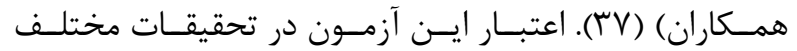

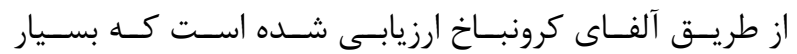

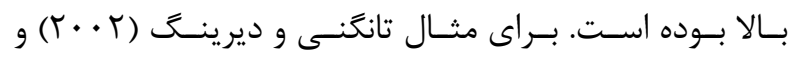

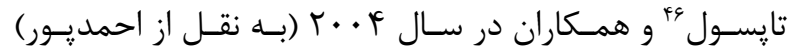

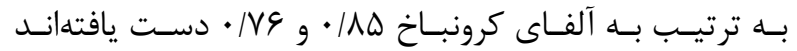
(YN)

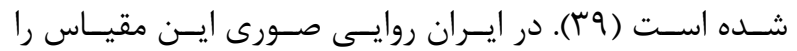

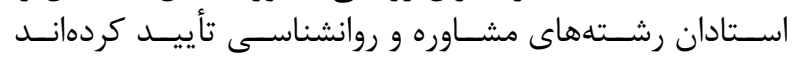
(YY)

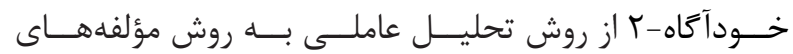

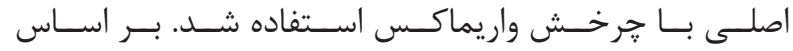

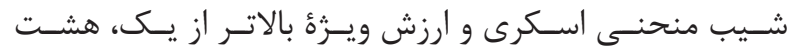

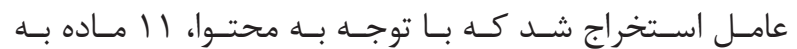

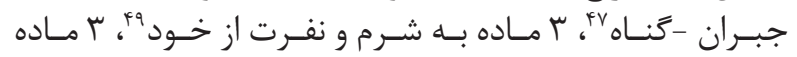

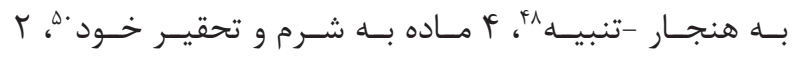

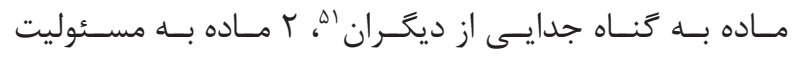

\footnotetext{
${ }^{30}$ Luminet

${ }^{31}$ Brooding

${ }^{32}$ Reflection

${ }^{33}$ Self-compassion scale

${ }^{34}$ convergent validity

${ }^{35}$ discriminant validity

${ }^{36}$ Self-empathy

${ }^{37}$ Self-kindness

${ }^{38}$ Self-isolation

${ }^{39}$ Common humanity/mindfulness

${ }^{40}$ Mindful attention awareness scale
}

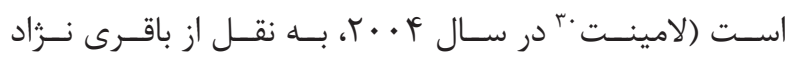

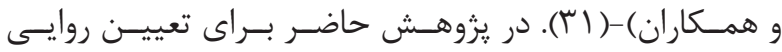

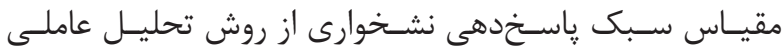

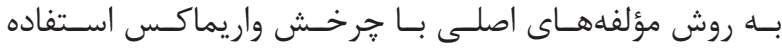

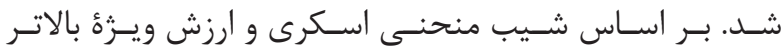

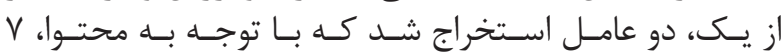

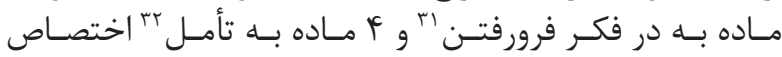

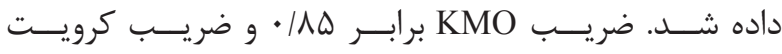

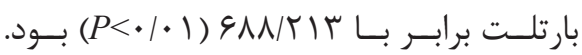

يرسشنامهُ شفقت خود

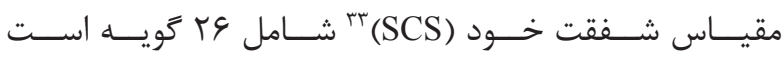

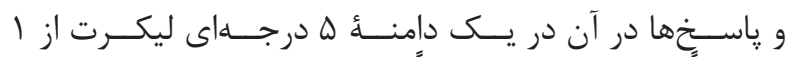

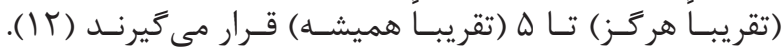

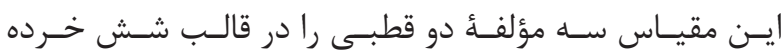

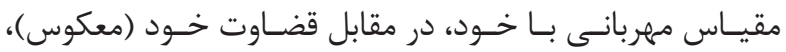

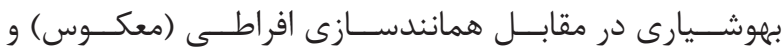

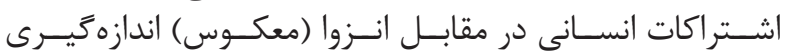

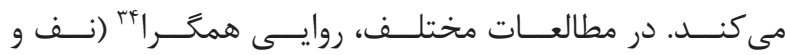

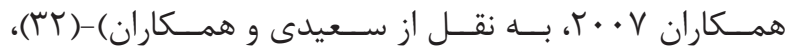

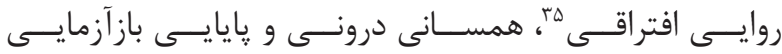

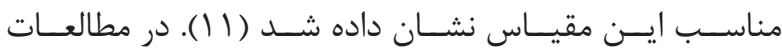

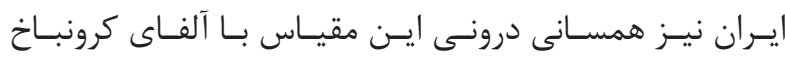

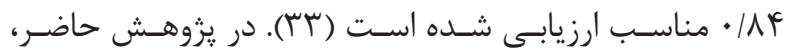

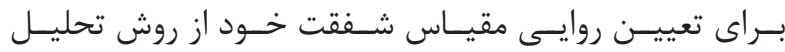

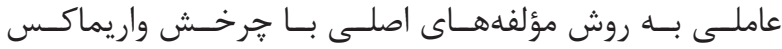

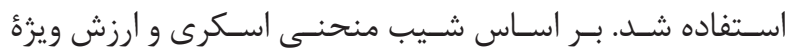

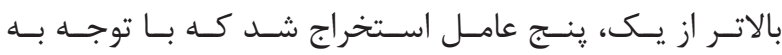

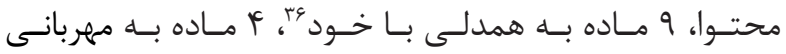

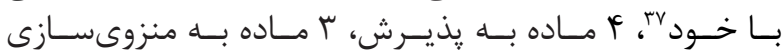

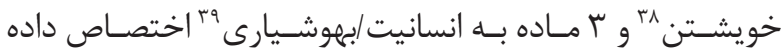

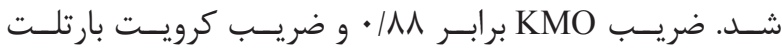

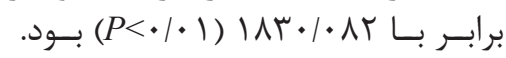

يرسشنامةُ بهوشيارى

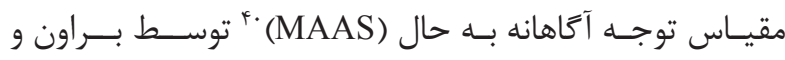

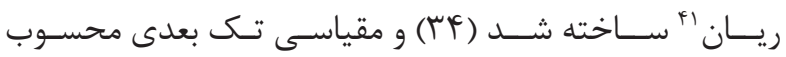

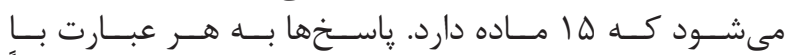

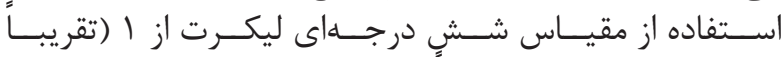

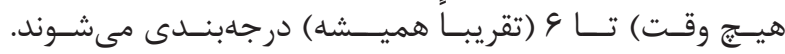

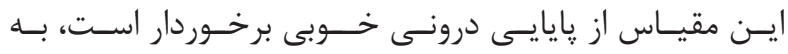
${ }^{41}$ Brown and Ryan
${ }^{42}$ Acting with awareness
${ }^{43}$ Observing ${ }^{44}$ Centralization
${ }^{45}$ Test of self-conscious affect
${ }^{46}$ Tapsol
${ }^{47}$ Repaire-quilt
${ }^{48}$ Shame and self -hate
${ }^{49}$ Normative-punitive shame
${ }^{50}$ Shame and self -humiliation
${ }^{51}$ Quilt of separation of others 
جدول r - ضرايب ركرسيونى شرم با بهوشيارى و شفقت خود.

\begin{tabular}{|c|c|c|c|}
\hline سطح معنى دارى & $\mathrm{t}$ & ضريب استاندارد شده ß & 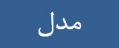 \\
\hline$\cdot / 1 \cdot 4$ & אس &.$- / .94$ & بهوشيارى \\
\hline.$/ . .1$ & -G/Arr & $-\cdot /$ rAS & شفقت خود \\
\hline
\end{tabular}

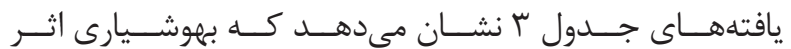

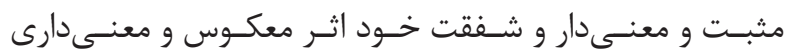

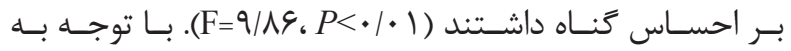

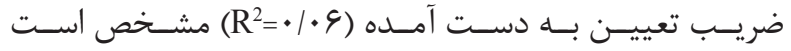

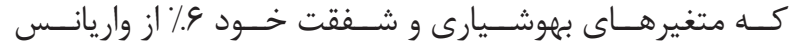

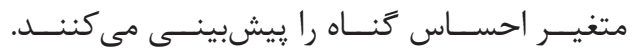
جدول r - ضرايب رَرسيونى گَناه با بهوشيارى و شفقت خود.

\begin{tabular}{|c|c|c|c|}
\hline سطح معنى دارى & $t$ & ضريب استاندارد شده & مدل \\
\hline.$/ . .1$ & $r / I V q$ & . $/ T \Delta T$ & بهوشيارى \\
\hline$\cdot 1 \cdot r$ & $-r / 9 r \Lambda$ &.$- / I V V$ & شفقت خود \\
\hline
\end{tabular}

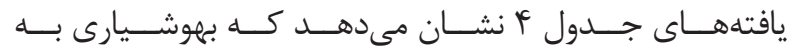

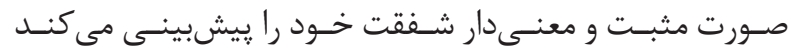

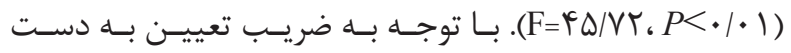

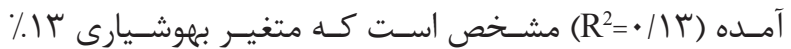

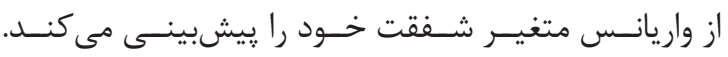

جدول F- ضريب ركرسيونى شفقت خود با بهوشيارى.

\begin{tabular}{|c|c|c|c|}
\hline سطح معنى دارى & $\mathrm{t}$ & ضريب استاندارد شده ß & مدل \\
\hline$\cdots+1 \cdot 1$ & G/VGT &.$/ 490$ & بهوشيارى \\
\hline
\end{tabular}

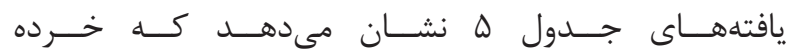

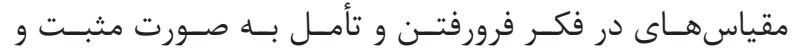

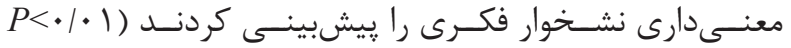
. (F=trVqRV/t\&T。

جدول هـ- ضرايب ركرسيونى نشخوار فكرى با خرده مقياسهاى نشخوار فكرى.

\begin{tabular}{|c|c|c|c|}
\hline سطح معنى دارى & $\mathrm{t}$ & ضريب استاندارد شده ß & مدل \\
\hline.$/ .1$ & fq1/Vre & ./VOT & در فكر فرورفتن \\
\hline 01 & $r \cdot 1 / 0 \cdot q$ &.$|49|$ & تأمل \\
\hline
\end{tabular}

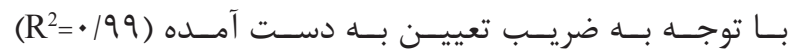

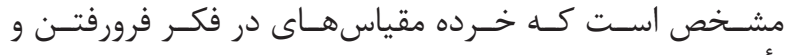

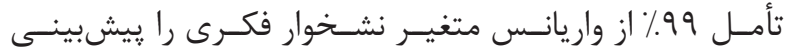

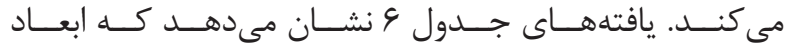

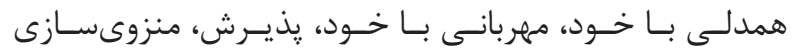

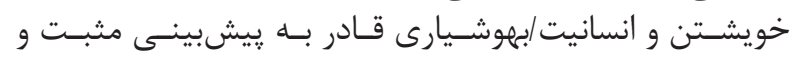

جدول 9- ضرايب ركرسيونى شفقت خود با خرده مقياسهاى شفقت خود.

\begin{tabular}{|c|c|c|c|}
\hline سطح معنى دارى & t & ضريب استاندارد شده & 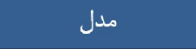 \\
\hline$\cdot 1 \cdots 1$ & FVG/TTD & .1011 & همدلى با خود \\
\hline $.1 . .1$ & TrH/AFr & . / TAF & مهربانى با خود \\
\hline.$/ \cdots 1$ & THV/FIV & . /TrA & يذيرش \\
\hline$\cdot 1 \cdots 1$ & $\mid \Lambda V / I Q \Lambda$ & .1194 & منزوىسازى خويشتن \\
\hline$=1 \cdots 1$ & $191 / \mathrm{VA}$ &.$/$ IVF & انسانيت لههوشيارى \\
\hline
\end{tabular}

${ }^{52}$ Responsibility-wrongdoing

${ }^{53}$ Conscience-quilt

${ }^{54}$ Shame and passive avoidance

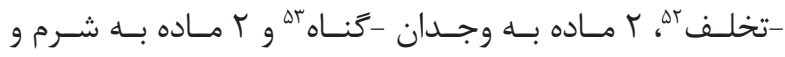

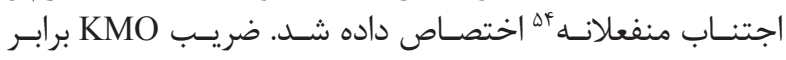

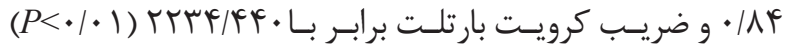

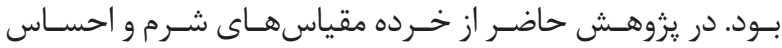

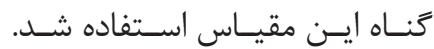

تجزيه و تحليل دادهها

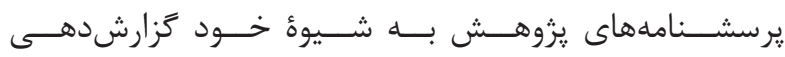

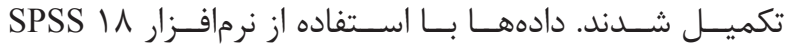

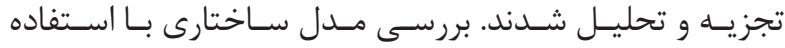

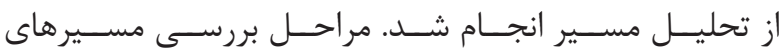

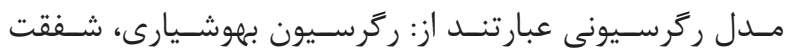

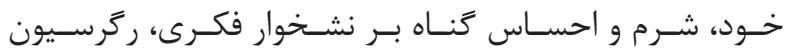

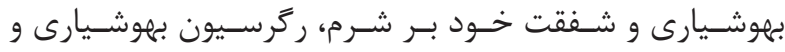

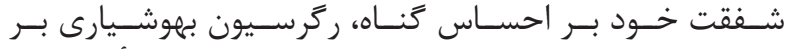

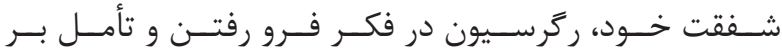

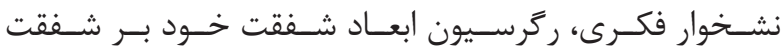

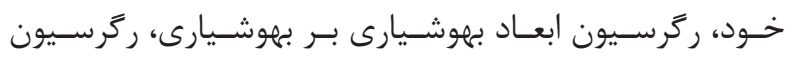

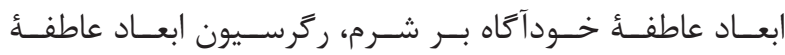

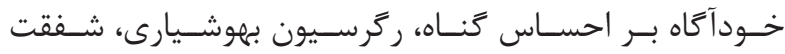

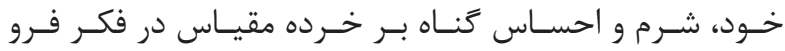

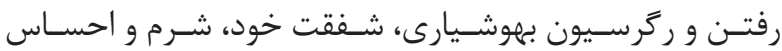

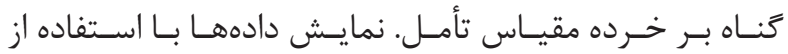

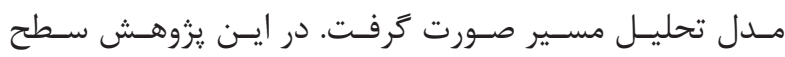

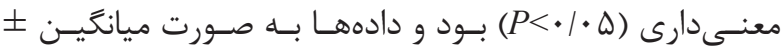

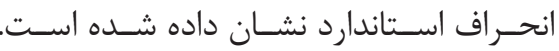

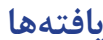

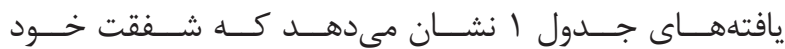

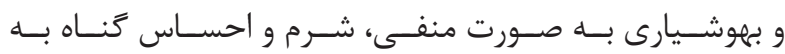

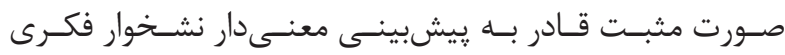

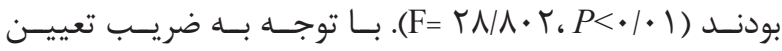

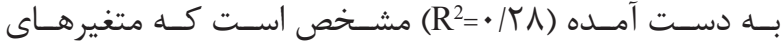

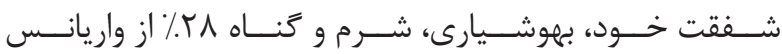

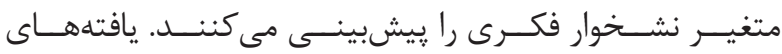

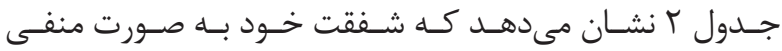

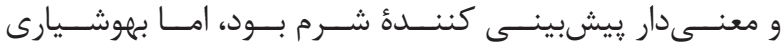

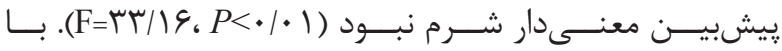

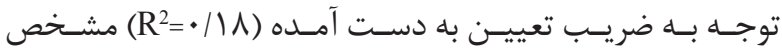

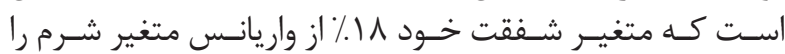

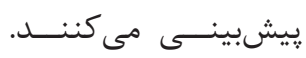
جدول ا- ضرايب ركر سيونى نشخوار فكرى با شفقت خود، بهوشيارى، شرم وكناه.

\begin{tabular}{|c|c|c|c|}
\hline سطح معنىدارى & $\mathrm{t}$ & ضريب استاندارد شده ß & مدل \\
\hline$\cdot$ & $-\omega / 9 V T$ &.$- / T F I$ & شفقت خود \\
\hline$\cdot / \cdots \wedge$ & $-r / 891$ & $-\cdot / 1$ \&A & بهوشيارى \\
\hline.$/ r r$ & $r /|F|$ &.$/ N F$ & شرم \\
\hline$\approx a^{*} \cdot 1 \cdot \cdots 1$ & T/TYT & $\cdot / \Lambda$. & كناه \\
\hline
\end{tabular}


جدول 9- ضرايب رَرسيونى كَناه با خرده مقياسهاى مقياس عاطفهُ خودآكاه -r.

\begin{tabular}{|c|c|c|c|}
\hline سطح معنى دارى & $t$ & ضريب استاندارد شده & مدل \\
\hline.$/ \cdots 1$ & $9 N / 94$. & - /AVG & جبران -كناه \\
\hline.$/ 119$ & 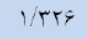 &.$/ \cdot 1 \mathrm{~V}$ & شرم و نفرت از خود \\
\hline.$/ \cdots 1$ & $N / 919$ & $\cdot / 1 \cdot 0$ & هنجار تنبيه \\
\hline$\cdot / \pi q$. & $\cdot / \Lambda 9$. &.$/ \cdot 1 \mathrm{~T}$ & شرم و تحقير خود \\
\hline.$/ \cdots 1$ & N/FET &.$/ .91$ & كَتاه جدايى از ديكران \\
\hline . RAt & $\cdot / v r$. & .1 .1$. & مسئوليت -تخلف \\
\hline$\cdot / \cdots 1$ & $N / 990$ &.$/|T|$ & وجدان -كناه \\
\hline$e^{* 1}$ & $-4 / T \& 9$ & $-\cdot / \cdot \Delta \varphi$ & شرم و اجتناب منفعلانه \\
\hline
\end{tabular}

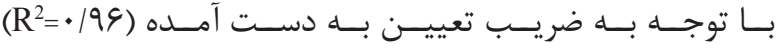

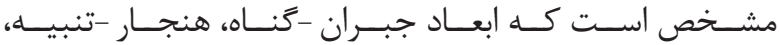

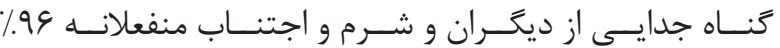

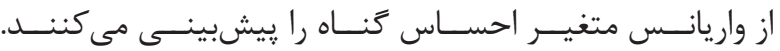

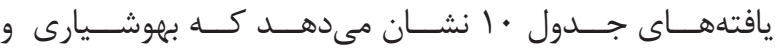

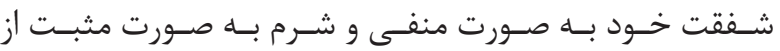

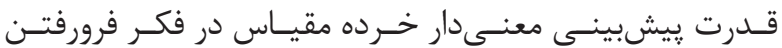

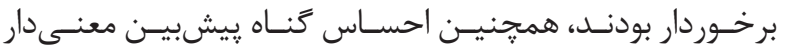

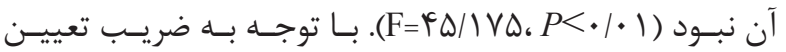

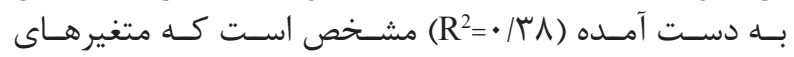

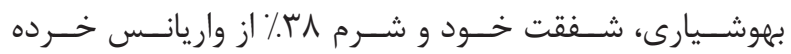

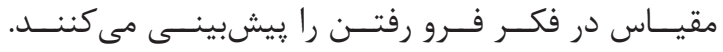
جدول • ا- ضرايب ركرسيونى خرده مقياس در فكر فرورفتن با شفقت خود، بهوشيارى،

\begin{tabular}{|c|c|c|c|}
\hline سطح معنى دارى & $\mathrm{t}$ & ضريب استاندارد شده & مدل مدل \\
\hline.$/ \ldots 1$ & WTKKT &.$- / 1 \vee G$ & بهوشيارى \\
\hline.$/ .1$ & $-N / \Delta \psi \Delta$ &.$- / F \Delta r$ & شفقت خود \\
\hline.$/ .14$ & $r / F \in V$ & מr/ & شرم \\
\hline . MGST &.$/ 91 T$ & .1 .49 & كناه \\
\hline
\end{tabular}

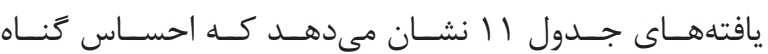

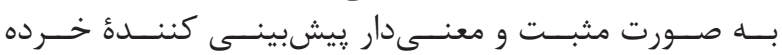

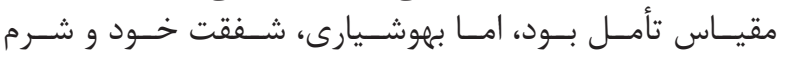

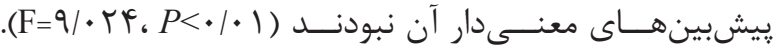

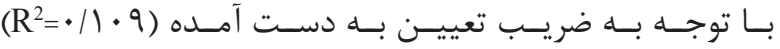

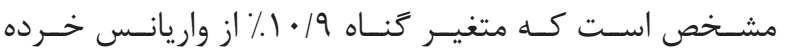

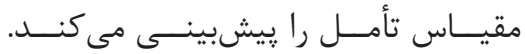
جدول Iال- ضرايب ركرسيونى خرده مقياس تأمل با بهوشيارى، شفقت خود، شرم و كناه.

\begin{tabular}{|c|c|c|c|}
\hline سطح معنى دارى & $\mathrm{t}$ & ضريب استاندارد شده & مدل \\
\hline.$/ 919$ &.$- / 491$ &.$- / . \mu_{1}$ & بهوشيارى \\
\hline.$/ 9 \vee 9$ &.$- / .4 q$ &.$- / .+r$ & شفقت خود \\
\hline . MYT & $\cdot / \lambda \cdot \Delta$ &.$/ . \Delta T$ & شرم \\
\hline .1 .1 & $0 / 1 \wedge 9$ &.$/ \pi 1 \pi$ & كناه \\
\hline
\end{tabular}

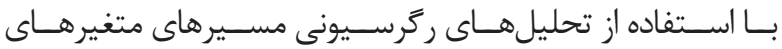

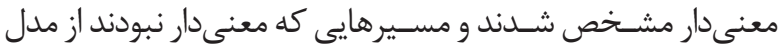

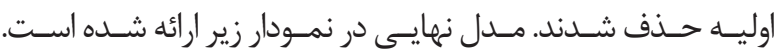

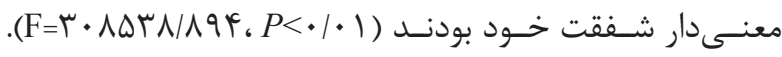

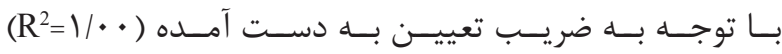

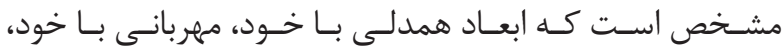

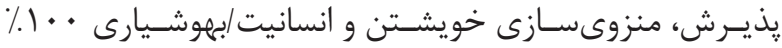

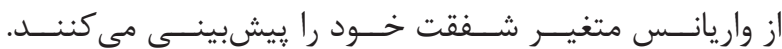

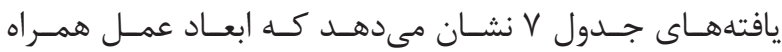

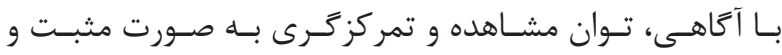

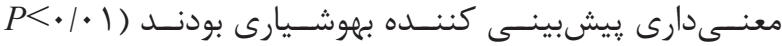
. $(F=) \omega \cdot r / T \cdot r$.

جدول V- ضرايب ركرسيونى بهوشيارى با خرده مقياسهاى بهوشيارى.

\begin{tabular}{|c|c|c|c|}
\hline سطح معنى دارى & t & ضريب استاندارد شده B & 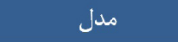 \\
\hline$\cdot 1 \cdot 1$ & $q \vee / 90$. & . $N \Delta A$ & عمل همراه با آكاهى \\
\hline.$/ .1$ & $1911 \%$ & . ITAT & توان مشاهده \\
\hline 0.1 .1 & $191 \Delta \Delta$. &.$/ F F s$ & تمركز كرى \\
\hline
\end{tabular}

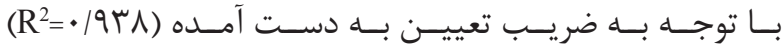

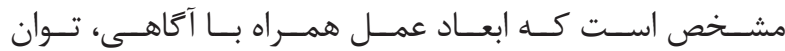

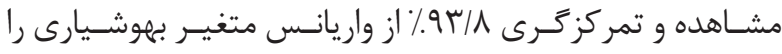

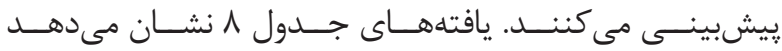

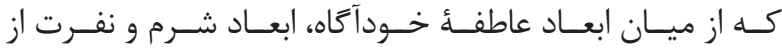

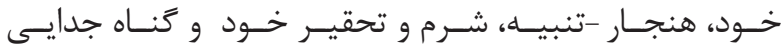

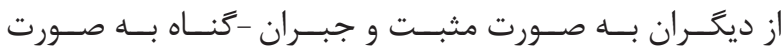

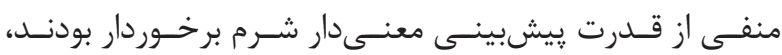

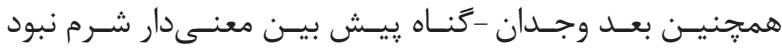

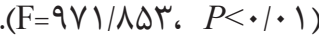

جدول ^- ضرايب ركرسيونى شرم با خرده مقياسهاى مقياس عاطفئ خودآكاه -r.

\begin{tabular}{|c|c|c|c|}
\hline سطح معنى دارى & $\mathrm{t}$ & ضريب استاندارد شده ß & 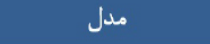 \\
\hline$\cdot / \cdots 1$ & $-\Delta / r \& 4$ & $-\cdot / \cdot v_{1}$ & جبران -كناه \\
\hline$\cdot \cdot \cdots 1$ & $r \Delta / \Delta \Lambda 1$ & . / rrq & شرم و نفرت از خود \\
\hline$\cdot \cdot \cdots 1$ & $1 N / q \cdot V$ & $\cdot / r \mu \lambda$ & هنجار تنبيك \\
\hline$\cdot \cdots 1$ & rN/9OH & $\cdot / 4 \cdot 9$ & شرم و تحقير خود \\
\hline$\cdot \cdot \cdot 1$ & $V / \Lambda 1 I$ &.$/ .94$ & كناه جدايى از ديخران \\
\hline$\cdot \cdots 1$ & $\mid f / \wedge \Delta F$ & $\cdot / T \cdot 1$ & مسئوليت -تخلف \\
\hline$\cdot / \mathrm{AN}$ &.$/ I F T$ & $\cdot / r$ & وجدان -خناه \\
\hline$\cdots 1$ & ID/VTV &.$/ T 19$ & شرم و اجتناب منفعالنه \\
\hline
\end{tabular}

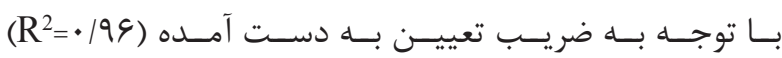

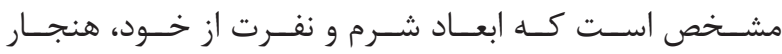

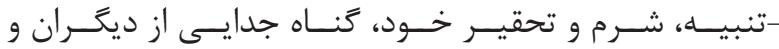

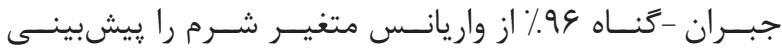

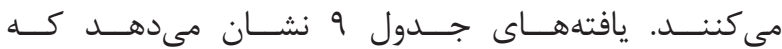

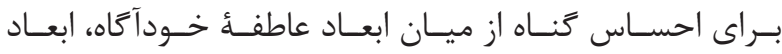

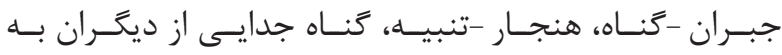

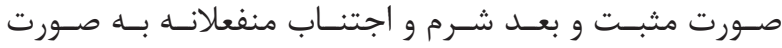

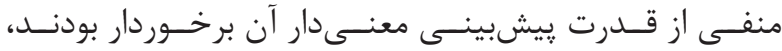

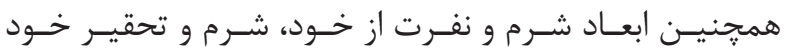

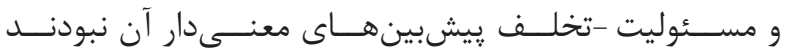

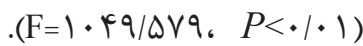




\section{بحث و نتيجه}

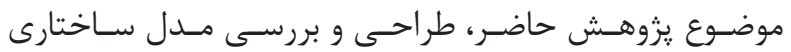

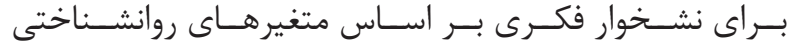

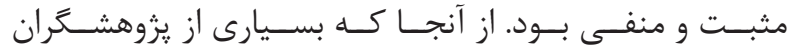

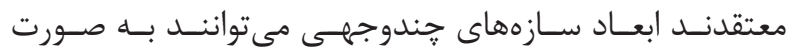

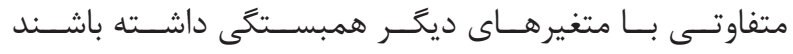

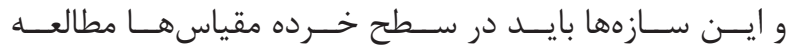

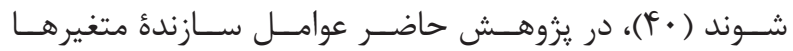

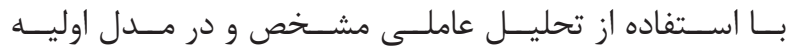

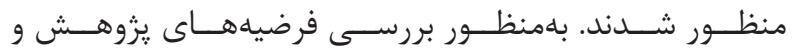

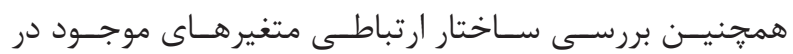

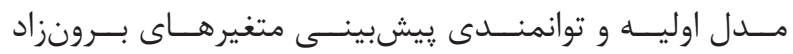

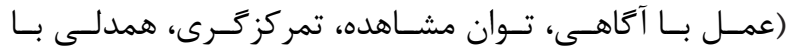

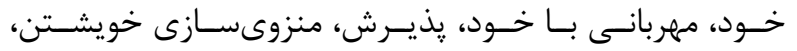

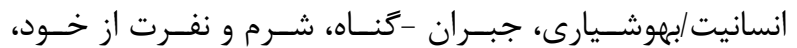

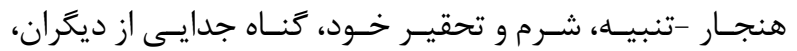

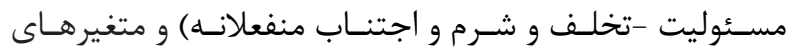

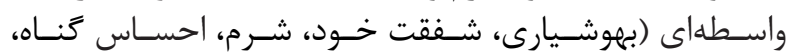

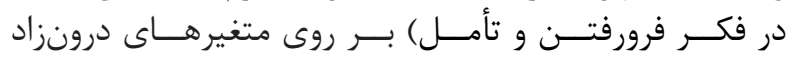

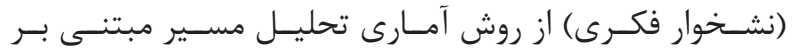

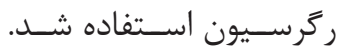

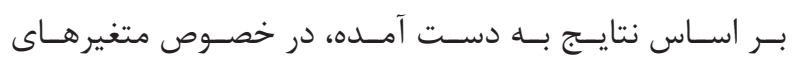

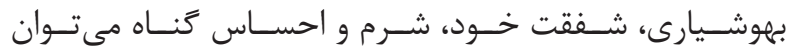

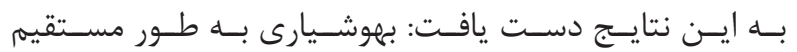

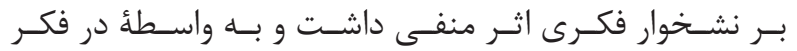

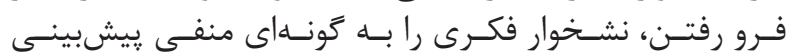

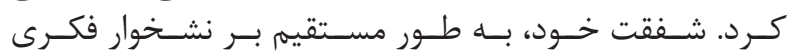

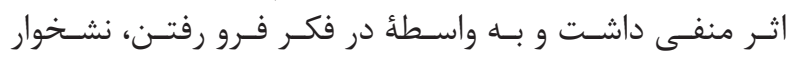

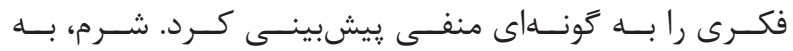

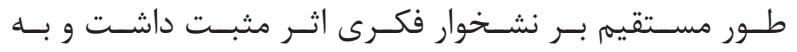

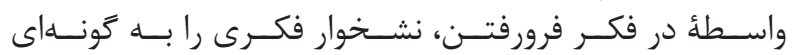

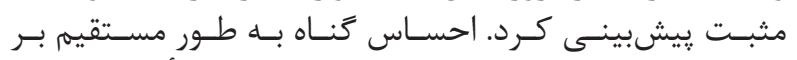

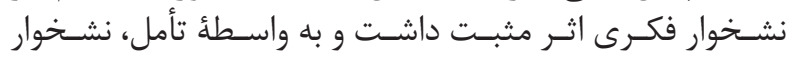

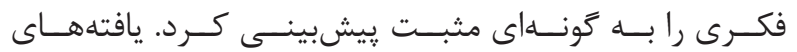

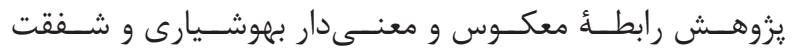

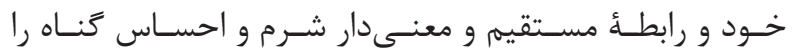

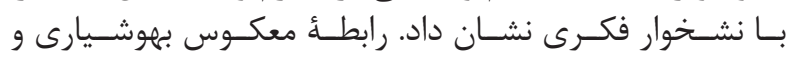

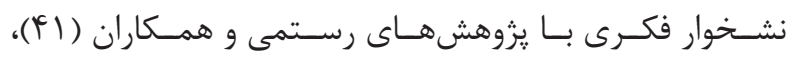

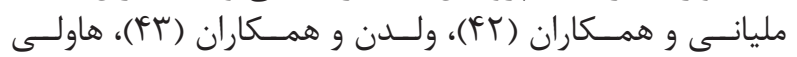

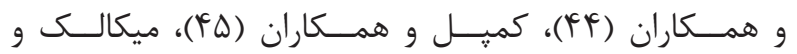

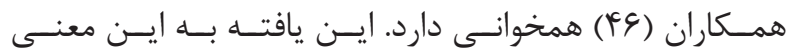

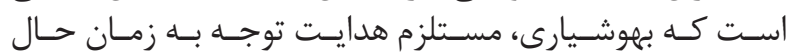

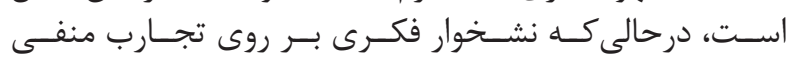

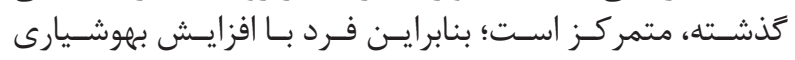

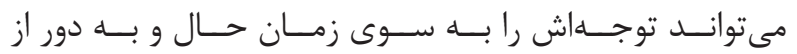

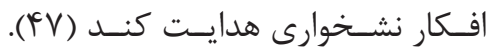

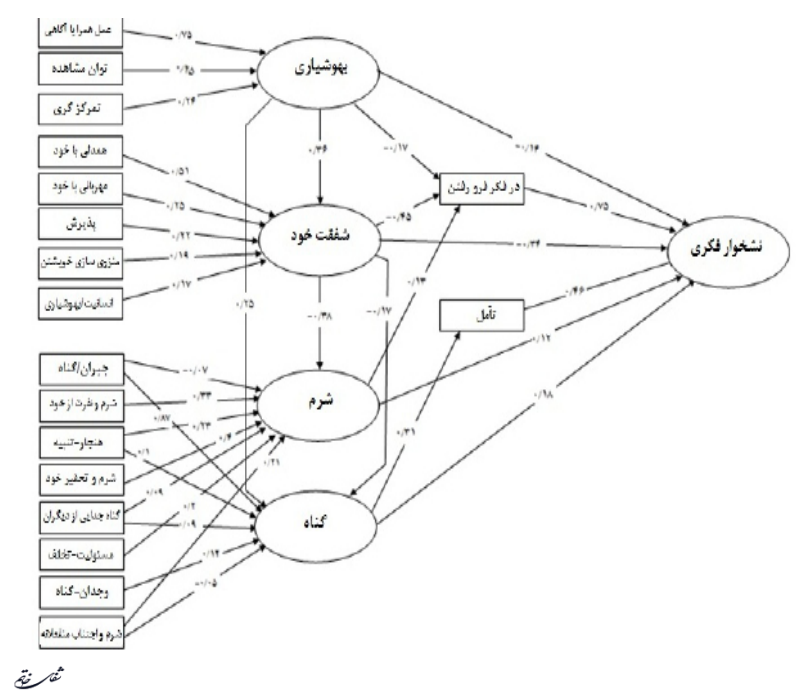

نمودار r - مدل نهايى نشخوار فكرى بر اساس متغيرهاى روانشناختى مثبت و منفى.

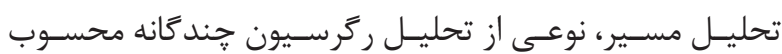

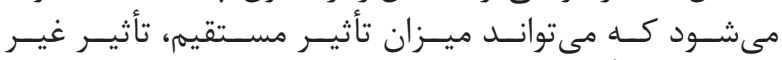

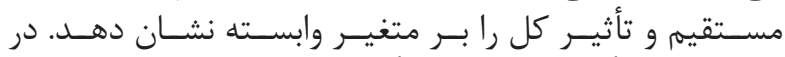

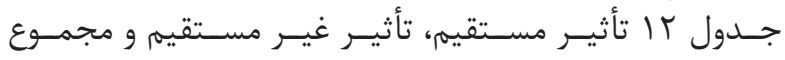

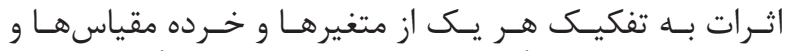

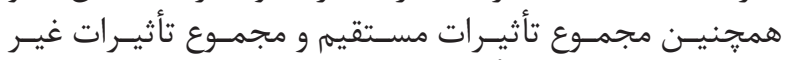

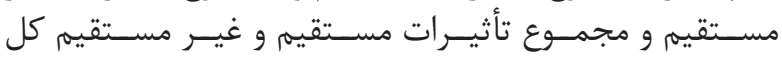

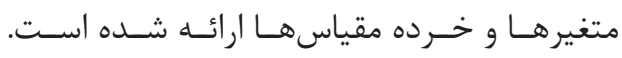

جدول rا - مجموع اثرات مستقيم و غير مستقيم بر نشخوار فكرى.

\begin{tabular}{|c|c|c|c|}
\hline مجموع اثرات & اثرات غير هستقيم & اثرات مستقيم & متغيرهاى مستقل \\
\hline.$- / \kappa \varepsilon$ &.$- / \pi T$ &.$- / 1 f$ & بهوشيارى \\
\hline.$- / \mathrm{vq}$ &..$/ 4 \Delta$ &.$- / \% F$ & شفقت خود \\
\hline.$/ \pi 1$ &.$/ .9$ &.$/ \mathrm{IT}$ & شرم \\
\hline.$/ 4 T$ &.$/ 14$ &.$/ 11$ & احساس كتاه \\
\hline.$/ \mathrm{YO}$ & - &.$/ \mathrm{VO}$ & خرده دفياس در فكر فرو رفتين \\
\hline.$/ 48$ & - &.$/ 49$ & خرده مقياس تأمل \\
\hline.$- / T T$ &.$- / T T$ & - & خرده مقياس عمل با أتاهي \\
\hline.$- / 11$ &.$- / 11$ & - & خرده مقياس توان مشاهده \\
\hline$-. \cdot / \cdot \Lambda$ &.$- / \cdot 1$ & - & خرده مقياس تمركزّرى \\
\hline$-\cdot / \Gamma \Lambda$ & $-\cdot / \Gamma \wedge$ & - & خرده مقياس همدلى با خود \\
\hline.$- / 11$ &..$/ 11$ & - & خرده مقياس مهربانى با خود \\
\hline.$- / 19$ &.$- / 19$ & - & خرده مقياس بذيرش \\
\hline.$- / 14$ &.$- / 11^{4}$ & - & خرده مقياس منزوى سازى خويشتن \\
\hline.$- / 11$ &.$- / 11$ & - & خرده مقياس انسانيت ابهوشيارى \\
\hline.$- / .14$ &.$- / .14$ & - & خرده مقياس جبران -كناه \\
\hline .1 .9 & .1 .9 & - & خرده مقياس شرم و نفرت از خود \\
\hline $.1 \%$ & $.1 \cdot 4$ & - & خرده هقياس هنجار -تنبيه \\
\hline$\cdot / \cdot \lambda$ & $.1 \cdot 1$ & - & خرده مقياس شرم و تحقير خود \\
\hline.$/ 1$ & $.1 \cdot 1$ & - & خرده مقياس كَّاه جدايي از ديكٔران \\
\hline.$\%$ &.$\%$ & - & خرده مقياس مسئوليت -تخلف \\
\hline.$/ * 4$ & .1 .4 & - & خرده مقياس شرم و اجتـاب منفعلاثه \\
\hline.$- V^{2}$ &.$- / 48$ &..$/ 11$ & جمع كل متغيرها \\
\hline
\end{tabular}




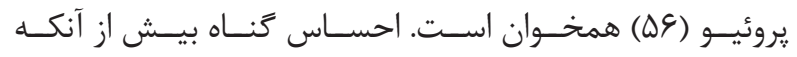

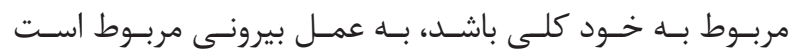

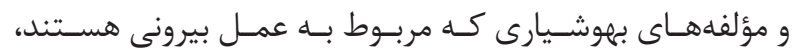

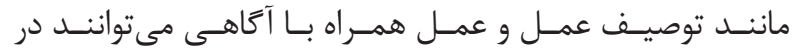

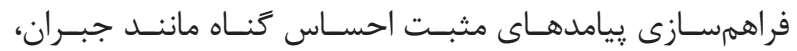

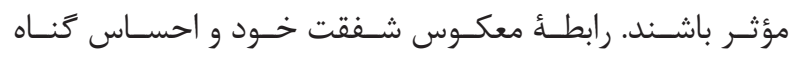

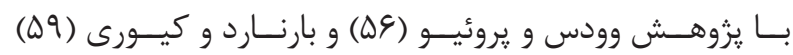

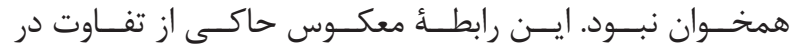

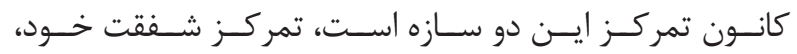

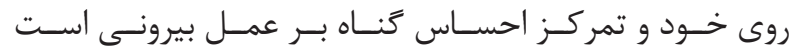

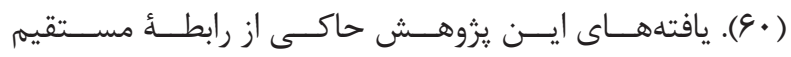

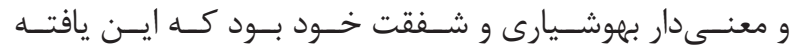

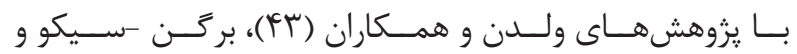

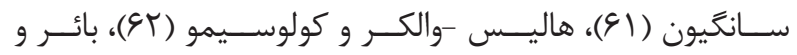

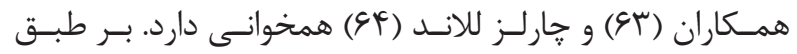

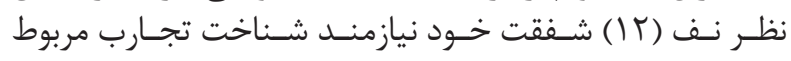

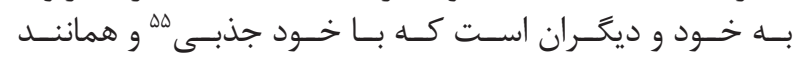

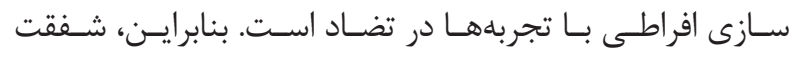

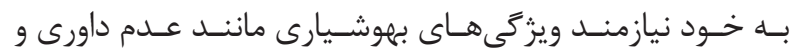

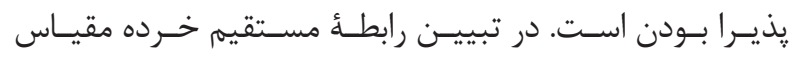

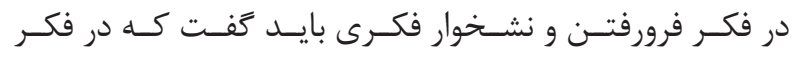

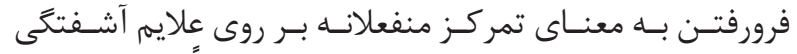

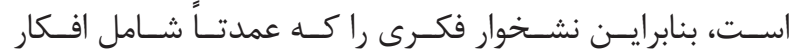

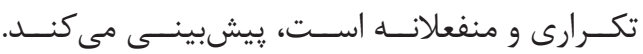

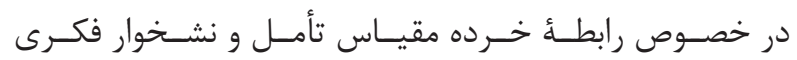

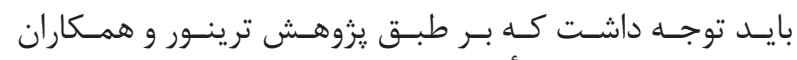

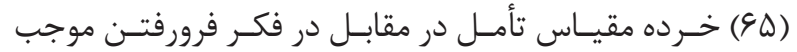

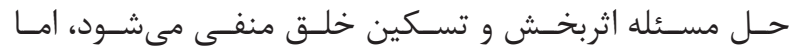

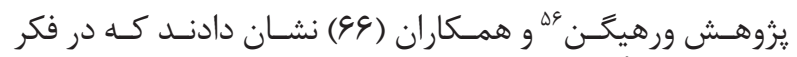

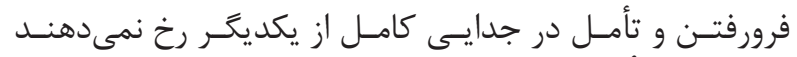

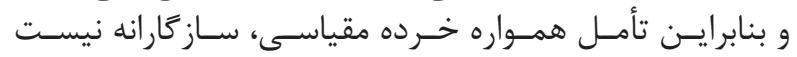

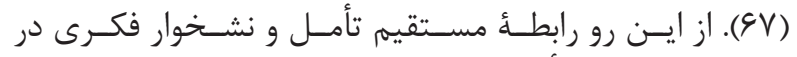

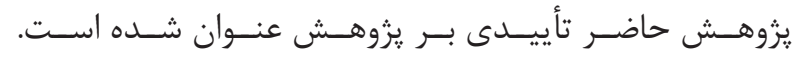

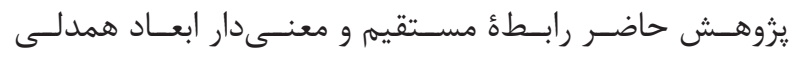

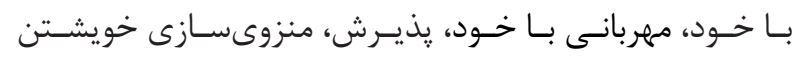

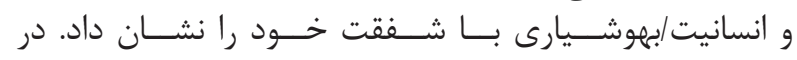

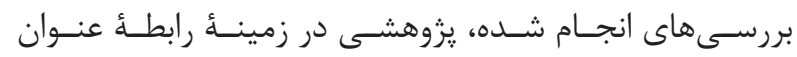

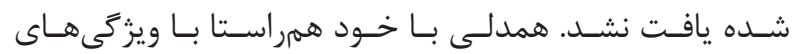

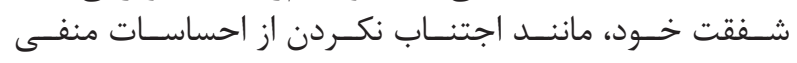

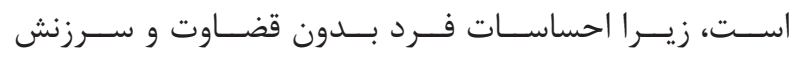

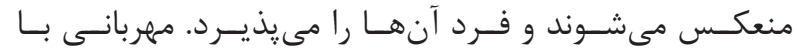

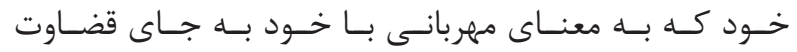

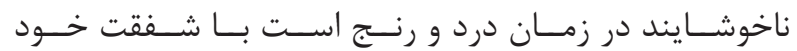

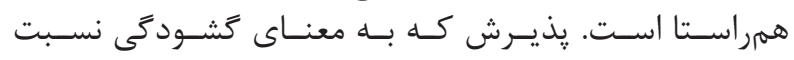

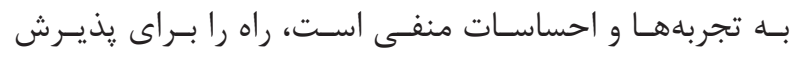

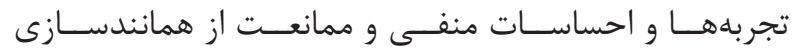

${ }_{55}^{5}$ Self-absorption

${ }^{56}$ Verhaegen

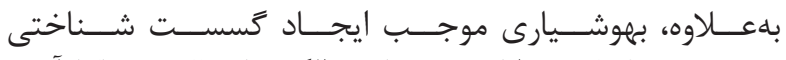

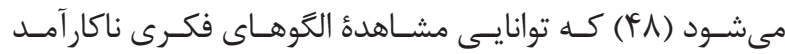

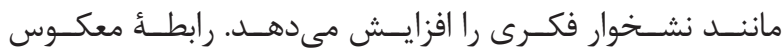

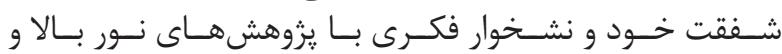

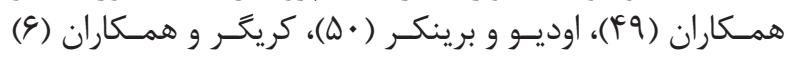

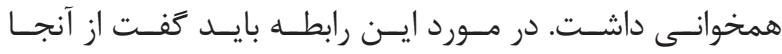

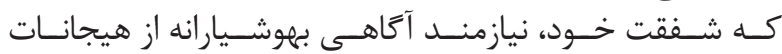

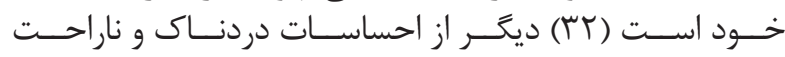

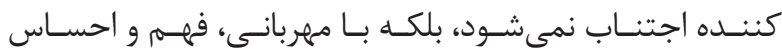

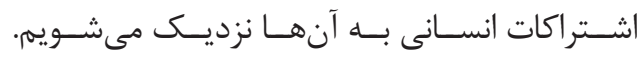

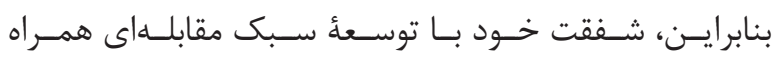

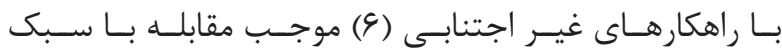

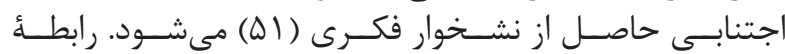

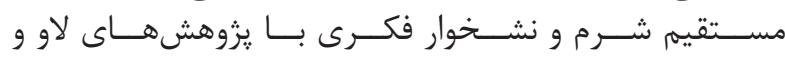

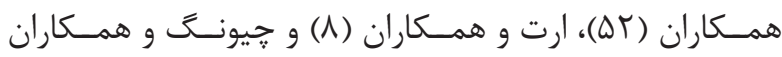

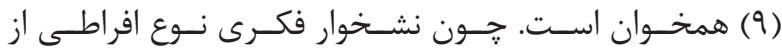

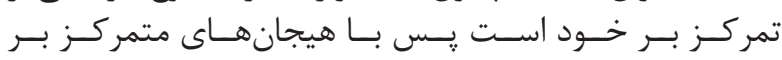

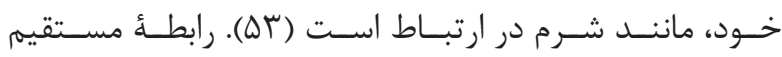

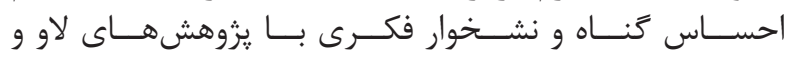

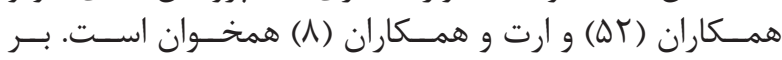

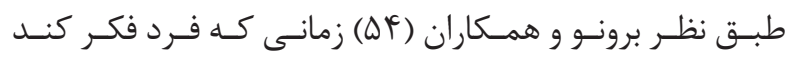

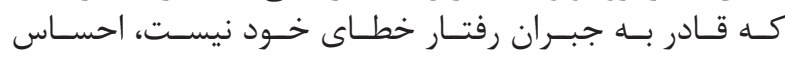

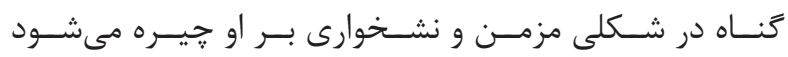

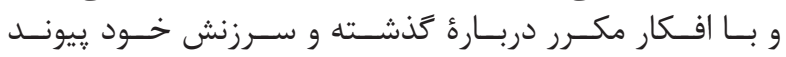

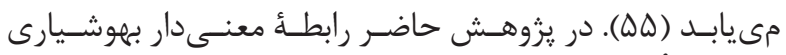

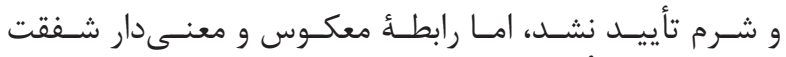

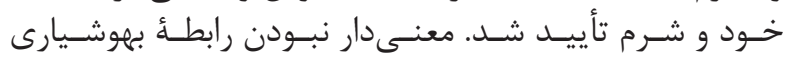

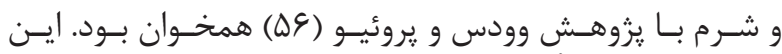

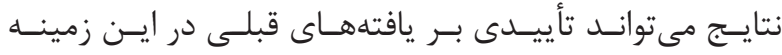

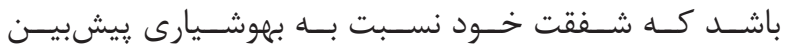

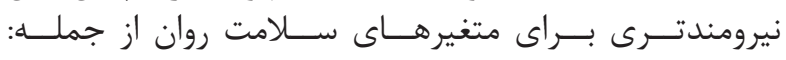

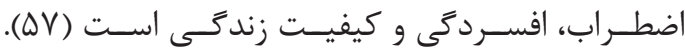

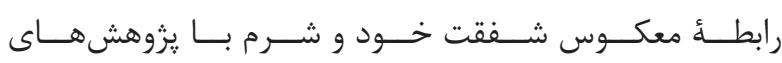

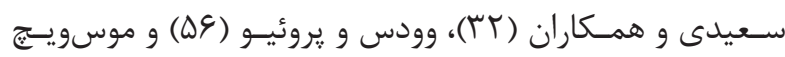

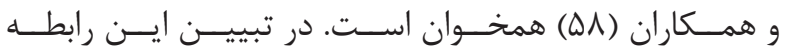

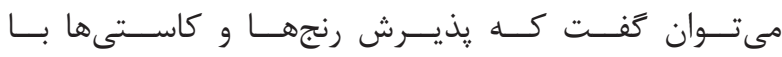

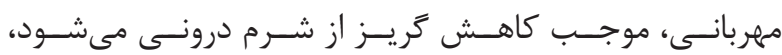

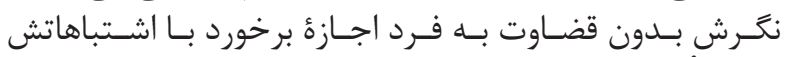

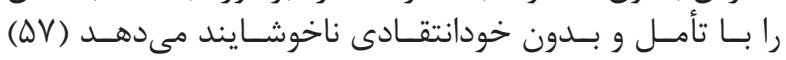

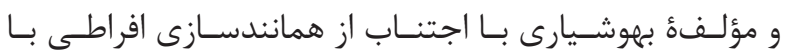

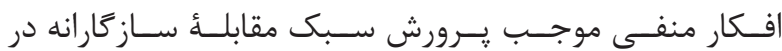

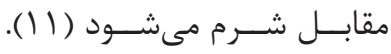

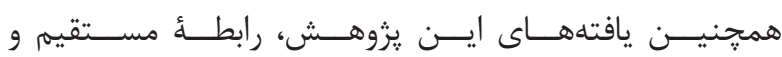

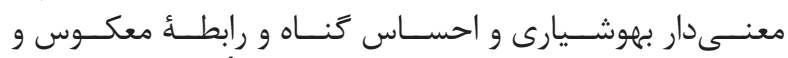

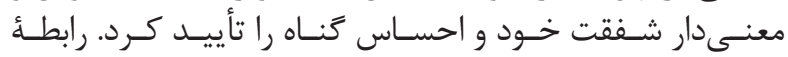

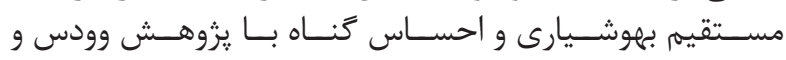




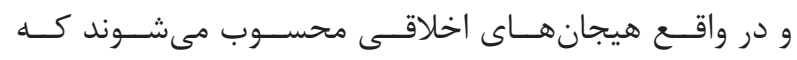

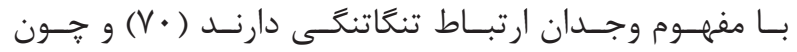

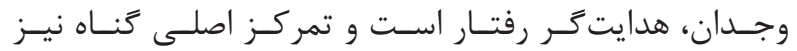

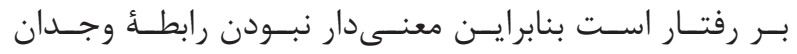

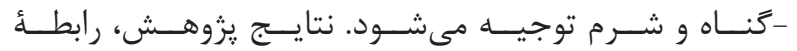

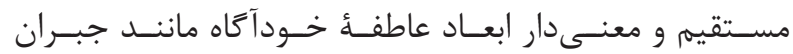

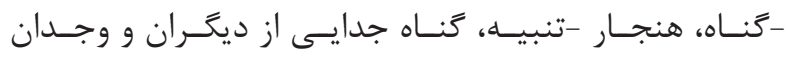

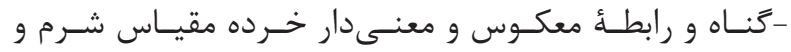

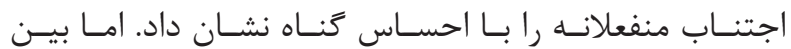

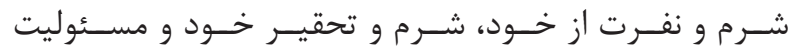

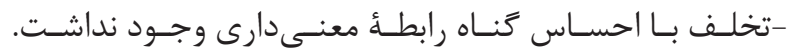

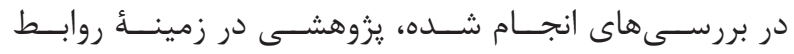

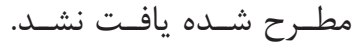

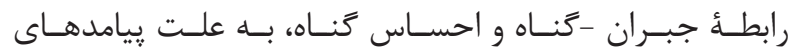

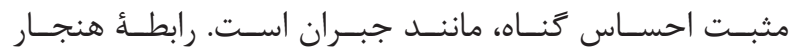

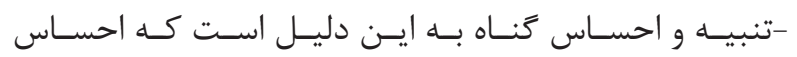

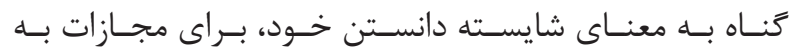

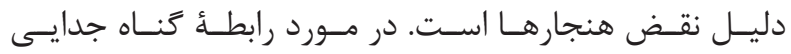

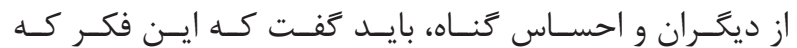

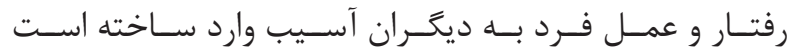

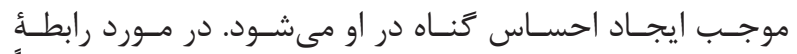

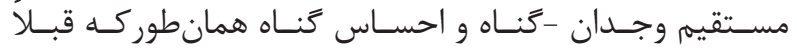

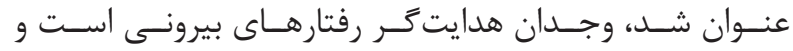

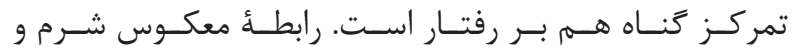

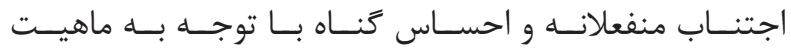

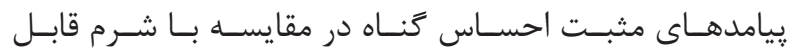
توجيــه اسـت

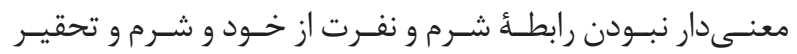

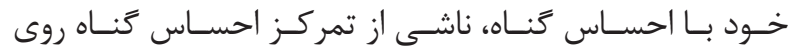

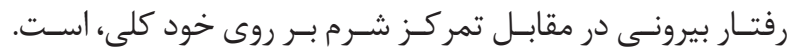

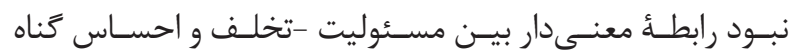

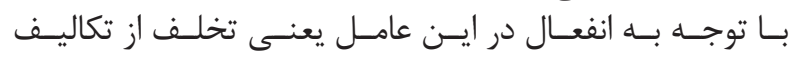

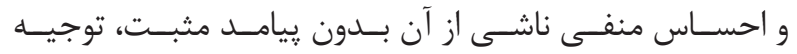

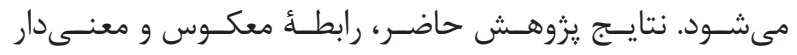

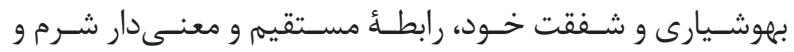

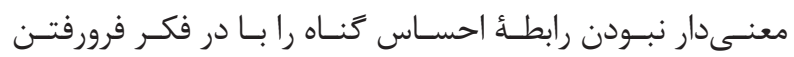

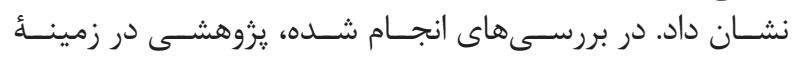

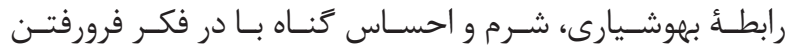

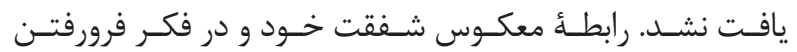

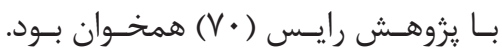

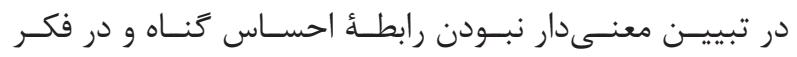

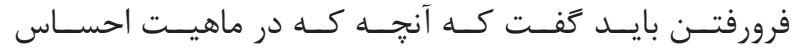

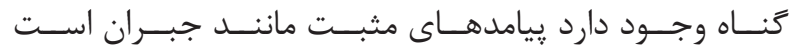

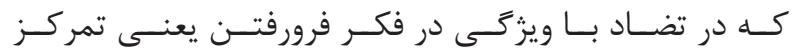

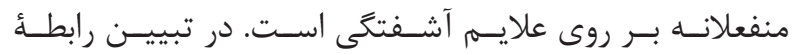

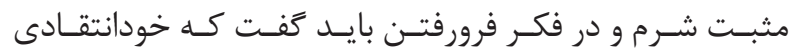

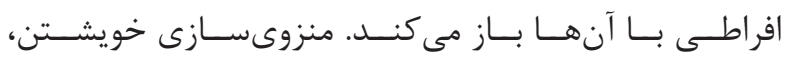

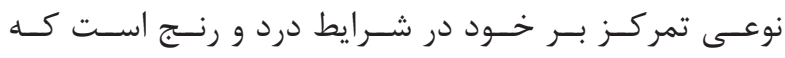

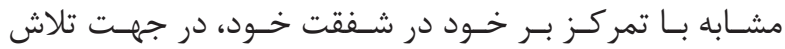

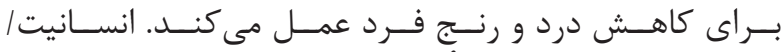

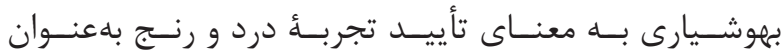

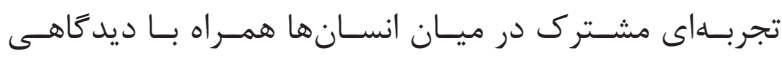

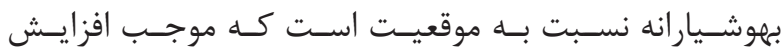

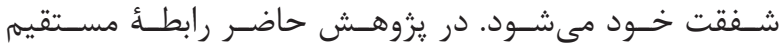

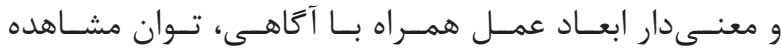

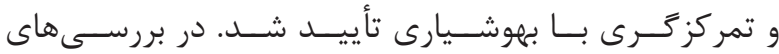

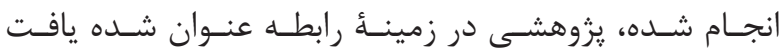

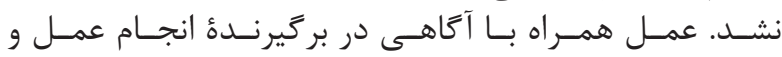

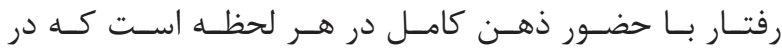

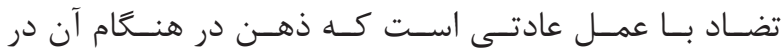

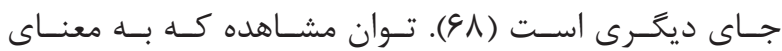

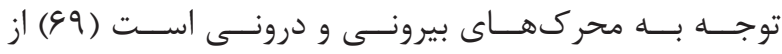

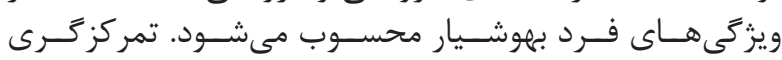

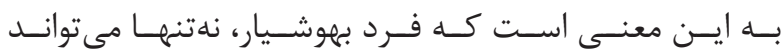

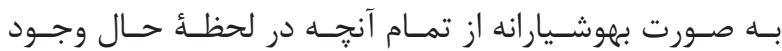

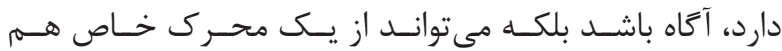

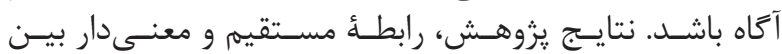

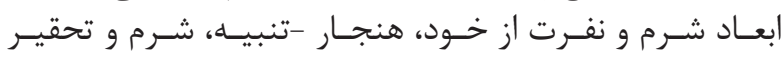

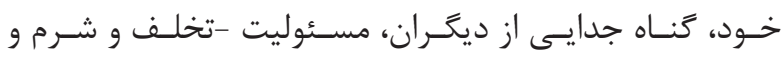

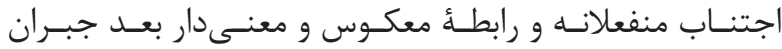

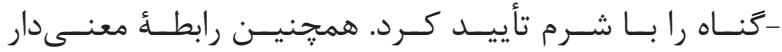

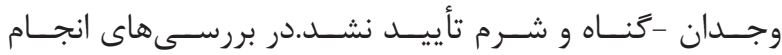

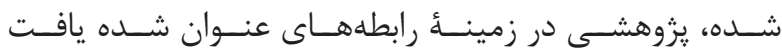

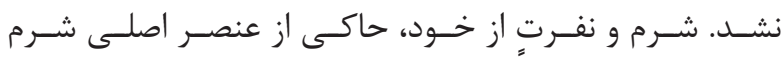

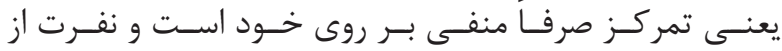

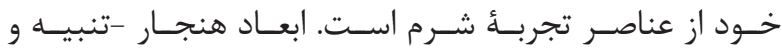

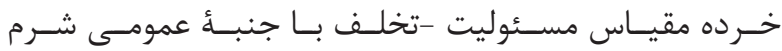

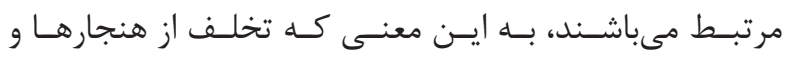

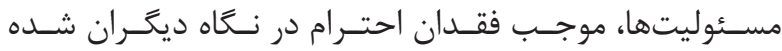

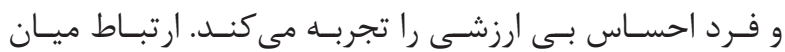

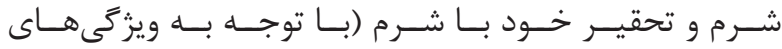

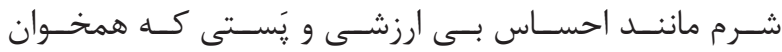

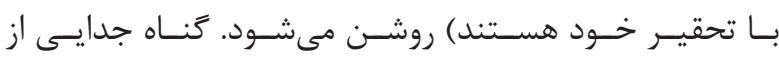

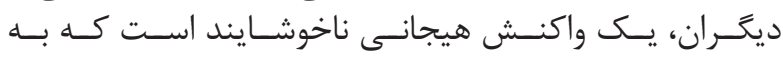

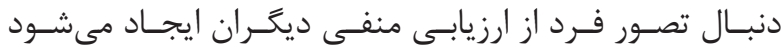

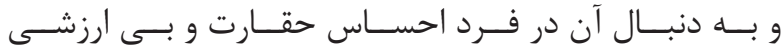

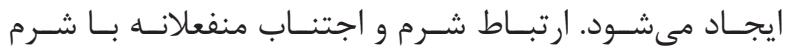

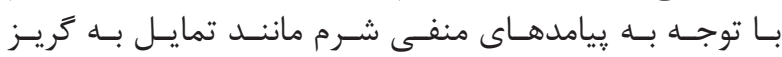

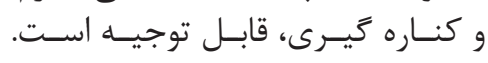

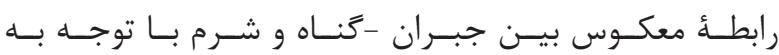

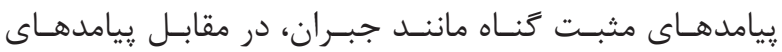

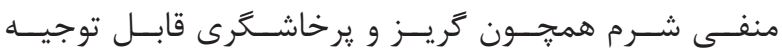

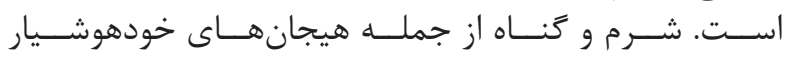




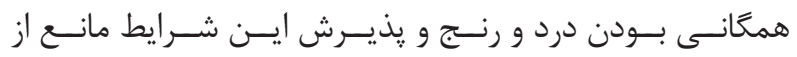

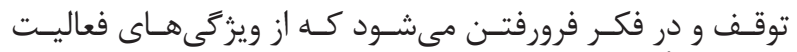

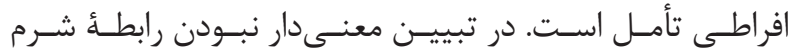

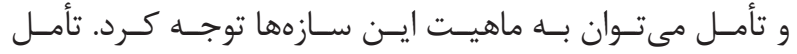

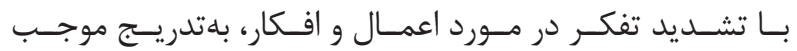

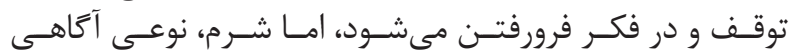

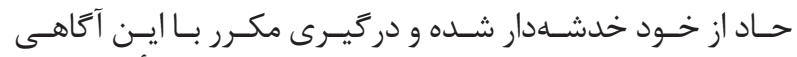

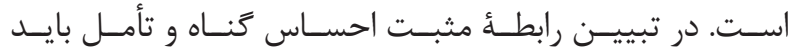

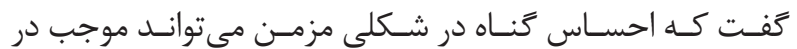

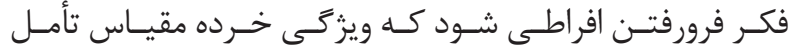

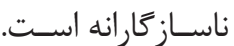

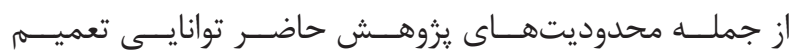

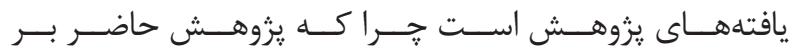

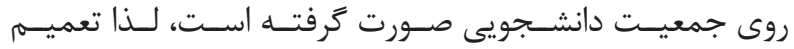

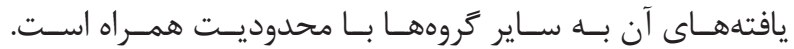

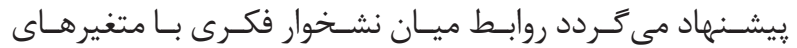

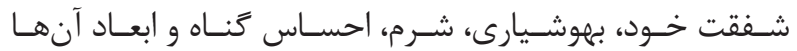

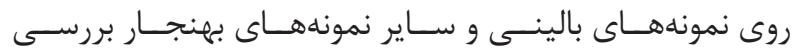

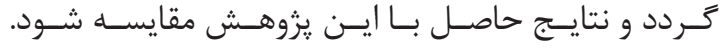

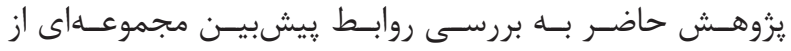

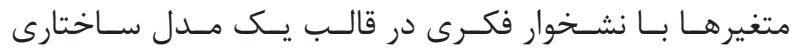

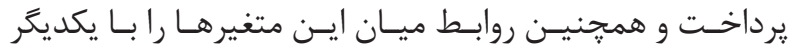

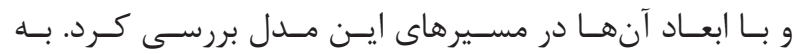

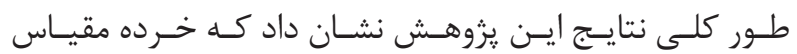

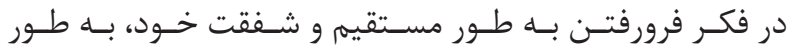

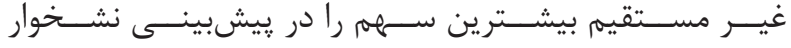
فكـرى داشـتند.

1. Yousefi Z. Rumination: definitions and theories. Psychotherapical Novelies. 2009; 51: 128-36.

2. Joormann J. Differentioal effect of rumination and dysphoria on the inhibition of irrelevant emotional material: evidance from a negative priming task. Cognit Ther Res. 2006; 30(2): 149-60.

3. Rippere V. "What's the thing to do when you're feeling depressed?" a pilot study. Behav Res Ther. 1977; 15(2): 185-91.

4. Nolen-Hoeksema S. Responses to depression and their effects on the durationof depressive episodes. J Abnorm Psychol. 1991; 100(4): 569-82.

5. Smith JM, Alloy LB. Aroadmap to rumination: a review of the definition, assessment, and conceptualization of this multifaceted construct. Clin Psychol Rev. 2009;

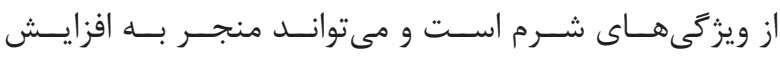

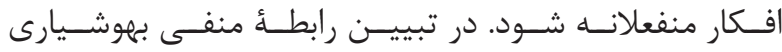

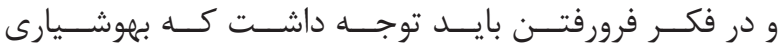

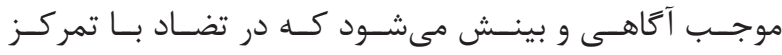

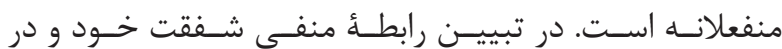

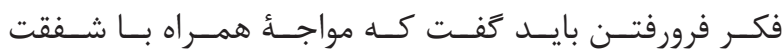

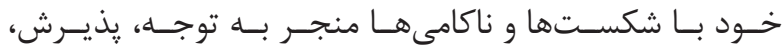

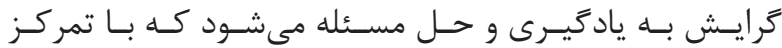

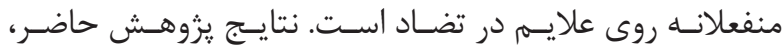

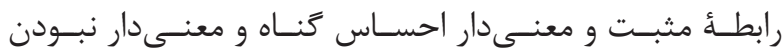

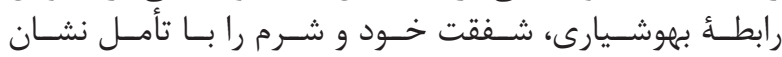

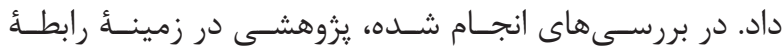

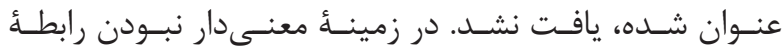

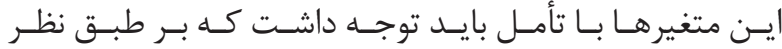

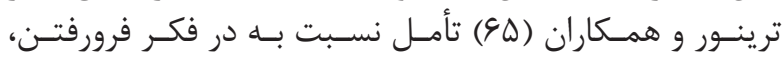

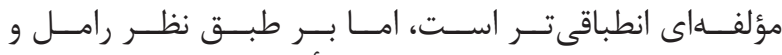

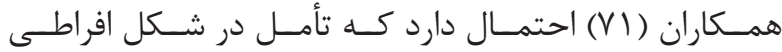

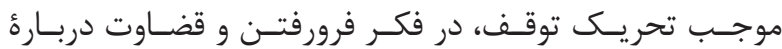

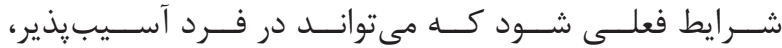

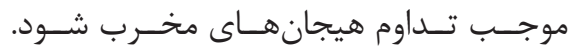

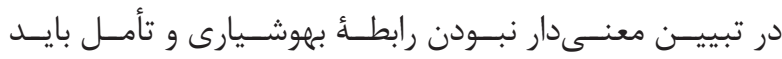

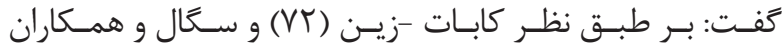

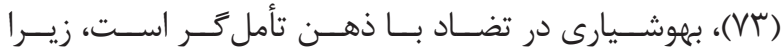

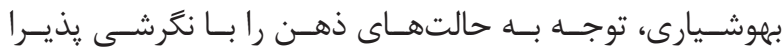

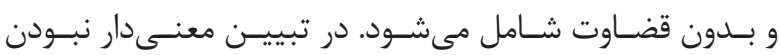

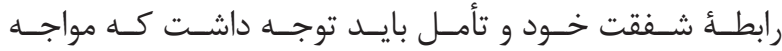

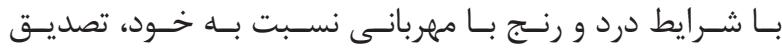

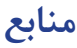

29(2): 116-28.

6. Krieger T, Altenstein D, Baettig I, Doerig N, Holtforth MG. Self-compassion in depression: associations with depressive symptoms, rumination, and avoidance in depressed outpatients. Behav Ther. 2013; 44(3): 501-13.

7. Coffey KA, Hartman M. Mechanisms of action in the inverse relationship between mindfulness and psychological distress. Complement Health Pract Rev. 2008; 13: 79-91.

8. Orth U, Berking M, Burkhardt S. Self-conscious emotions and depression: rumination explains why shame but not guilt is maladaptive. Pers Soc Psychol Bull. 2006; 32(12): 1608-19.

9. Cheung MS, Gilbert P, Irons C. An exploration 


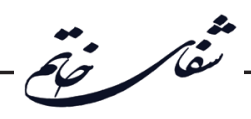

of shame, social rank and rumination in relation to depression. Pers Individ Dif. 2004; 36: 1143-53.

10. Manavipour D. A mathematical model to anticipate life satisfaction based on psychological wellbeing indices and irrational beliefs. Journal of Behavioral Sciences in Asia. 2014; 8: 84-91.

11. Neff KD. Self-compassion: an alternative conceptualization of a healthy attitude toward oneself. Self Identity. 2003; 2: 85-101.

12. Neff KD. The development and validation of a scale to measure self-compassion. Self Identity. 2003; 2: 22350.

13. Blatt SJ, Quinlan DM, Chevron ES, McDonald C, Zuroff D. Dependency and self-criticism: psychological dimensions of depression. J Consult Clin Psychol. 1982; 50(1): 113-24.

14. Wood JV, Saltzberg JA, Neale JM, Stone A. Selffocused attention, coping responses, and distressed mood in everyday life. J Pers Soc Psychol. 1990; 58: 1027-36.

15. Adams CE, Leary MR. Promoting selfcompassionate attitudes toward eating among restrictive and guilty eaters. J Soc Clin Psychol. 2007; 26: 1120-44.

16. Bishop SR, Lau M, Shapiro SL, Carlson L, Anderson N, Carmody J, et al. Mindfulness: a proposed operational definition. Clin Psychol Sci Pract. 2004; 11(3): 230-41.

17. Kabat-Zinn J. Mindfulness meditation: health benefits of ancient Buddhist practice. Goleman D, , Gurin J. Mind body medicine: how to use your mind for better health. New York: Consumer Reports Books: Yonkers. 1993; p. 262-3.

18. Baer RA. Mindfulness training as a clinical intervention: a conceptual and empirical review. Clin Psychol Sci Pract. 2003; 10: 125-43.

19. Miller JJ, Fletcher K, Kabat-Zinn J. Three year follow-up and clinical implications of a mindfulness meditation-based stress reduction intervention in the treatment of anxiety disorders. Gen Hosp Psychiatry. 1995; 17: 192-200.

20. Astin J. Stress reduction through mindfulness meditation: effects on psychological symptomatology, sense of control, and spiritual experiences. Psychother Psychosom. 1997; 66: 97-106.

21. Shapiro SL, Schwartz G, Bonner G. Effects of mindfulness-based stress reduction on medical and premedical students. J Behav Med. 1998; 21(6): 581-99.

22. Reibel DK, Greenson JM, Brainard GC, Rosenzweig S. Mindfulness based stress reduction and health-related quality of life in a heterogeneous patient population. Gen Hosp Psychiatry. 2001; 23: 183-92.

23. Speca M, Carlson LE, Goodey E, Angen MJ. A randomized wait-list controlled clinical trial: The effect of a mindfulness-based stress reduction 73 program on mood and symptoms of stress in cancer outpatients. Psychosom Med. 2000; 62: 613-22.

24. Tangney JP, Wagner PE, Gramzow R. Proneness to shame, proneness to guilt, and psychopathology. J Abnorm Psychol. 1992; 101(3): 469-78.

25. Tangney JP. Situational determinants of shame and guilt in young adulthood. Pers Soc Psychol Bull. 1992; 18: 199-206.

26. Tilghman-Osborne CE. The Relation Of quilt, shame, behavioral slf-blame, and Characterological selfblame to depression in dolescents over time. MS thesis. Vanderbilt University, Department of Psychology. 2007.

27. Woien SL, Ernst HAH, Patock-Peckham JA, Nagoshi CT. Validation of the TOSCA to measure shame and guilt. Pers Individ Dif. 2003; 35: 313-26.

28. Demehri F, Honarmand MM, Yavari AH. Depression and obsessive-compulsive as antecedent of guilt feeling and anxiety as consequences of it in university girl students. Journal of Women and Culture. 2011; 2(6): 25-36.

29. Kamrimian N, Karimi Y, Bahmani B. The relationship of mental health dimensions and guilt feeling with marital commitment in married people. Journal of Family Counseling \& Psychotherapy. 2011; 1(2): 243-56.

30. Nolen-Hoeksema S, Morrow J. A prospective study of depression and posttraumatic stress symptoms after a natural disaster: The 1989 Loma Prieta earthquake. J Pers Soc Psychol. 1991; 61(1): 115-21.

31. Bagherinezhad M, Salehi Fadardi J, Tabatabayi SM. The relationship between rumination and depression in a sample of Iranian student. Research in Clinical Psychology and Counselings. 2010; 11(1): 21-38.

32. Saeedi Z, Ghorbani N, Sarafraz MR, Sharifian $\mathrm{MH}$. The effect of inducing self-compassion and selfesteem on the level of the experience of shame and guilt. 
Contemporary Psychology. 2013; 8(1): 91-102.

33. Ghorbani N, Watson PJ, Zhuo C, Norballa F. Selfcompassion in Iranian Muslims:Relationships with Integrative self-knowledge,mental health, and religious orientation. Int J Psychol Relig. 2012; 22(2): 106-18.

34. Brown KW, Ryan RM. The benefits of being present: mindfulness and its role in psychological wellbeing. J Pers Soc Psychol. 2003; 84(4): 822-48.

35. Ghasemipour Y, Ghorbani N. Mindfulness and basic psychological needs among patients with coronary heart disease. IJPCP. 2010; 16(2): 154-62

36. Pourshahriari S. The Relationship between shame with dimensions of anger at University of Tehran. Quarterly Journal of Psychological Studies. 2009; 5(3): 27-46.

37. Anoshei M, Pourshahriyar MS, Sana'i Zakir B. The relationship between the perception of girls' educational models in their parents with a sense of shame and guilt. Counseling Research and Developments. 2008; 27(7): 7-26.

38. Ahmadpor A. Construction and standardization of shame and guilt questionnaire at state universities in Tehran. Master Thesis. Tehran. Allameh Tabatabai University. 2005.

39. Zarei S, Farahbakhsh K, Esmaeili M. The determination of the share of self-differentiation, trust, shame, and guilt in the prediction of marital adjustment. Knowledge and Research in Applied Psychology. 2011; 12(45): 51-63.

40. Smith GT, Fischer S, Fister SM. Incremental validity principles in test construction. Sychological Assessment. 2003; 15: 467-77.

41. Rostami A, Shariatnia K, Khajehvand Khoshli A. The relationship between self-efficacy and mind fullness with rumination among students of Islamic Azad University, Shahrood Branch. Medical Sciences. 2015; 24(4): 254-9.

42. Melyani M, Alahyari A, Azad Falah P, Fathi Ashtiani A, Tavoli A. Mindfulness based cognitive therapy versus cognitive behavioral therapy on residual symptoms in recurrent depression. Journal of Behavioral Sciences. 2013; 7(2): 17-8.

43. Velden AM, Kuyken W, Wattar U, Crane C, Pallesen KJ, Dahlgaard J, et al. A systematic review of mechanisms of change in mindfulness-based cognitive therapy in the treatment of recurrent major depressive disorder. Clin Psychol Rev. 2015; 37: 26-39.

44. Hawley LL, Schwartz D, Bieling PJ, Irving J, Corcoran K, Farb NAS, et al. Mindfulness practice, rumination and clinical outcome in mindfulness-based treatment. Cognit Ther Res. 2014; 38: 1-9.

45. Campbell TS, Labelle LE, Bacon SL, Faris P, Carlson LE. Impact of mindfulness-based stress reduction (mbsr) on attention, rumination and resting blood pressure in womenwith cancer: a waitlist-controlled study. J Behav Med. 2012; 35: 262-71.

46. Michalak J, Hölz A, Teisman T. Rumination as a predictor of relapse in mindfulness-based cognitive therapy for depression. Psychol Psychother. 2011; 84(2): 230-6.

47. Kiken LG. Looking up: mindfulness increases positive judgments and reduces negativity bias. Soc Psychol Personal Sci. 2011; 2425-31 :.

48. Germer CK. The mindful path to self-compassion: freeing your self from destructive thoughts and emotions. NewYork: Guilford. 2009.

49. Noorbal F, Borjali A, Noorbala AA. The interaction effect between "self compassion" and rumination" of depressed patients in "compassion focused therapy". Bimonthly Official Publication Medicine Daneshvar. 2013; 20(104): 77-84.

50. Odou N, Brinker J. Exploring the relationship between rumination, self-compassion, and mood. Self and Identity. 2014; 13(4): 449-59.

51. Liverant GI, Kamholz BW, Sloan DM, Brown TA. Rumination in clinical depression: a type of emotional suppression? Cognit Ther Res. 2011; 35: 253-65.

52. Law KC, Chapman AB, Alexander L. Borderline personality features as a potential moderator of the effect of anger and depressive rumination on shame, self-blame, and self- forgiveness. J Behav Ther Exp Psychiatry. 2015; 46: 27-34.

53. Reid RC, Temko J, Moghaddam JF, Fong TW. Shame, rumination, and self-compassion in men assessed for hypersexual disorder. J Psychiatr Pract. 2014; 20(4): 260-8.

54. Bruno S, Lutwak N, Agin M. Conceptualization of guilt and the corresponding relationship to emotional ambivalence, self-disclosure, loneliness and alienation. Personality and Individual Differences .2009; 47: 487-91. 


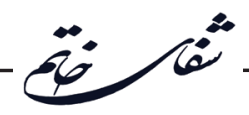

55. Lyubomirsky S, Tucker KL, Caldwell ND, Berg K. Why ruminators are poor problem solvers: Clues from the phenomenology of dysphoric rumination. J Pers Soc Psychol. 1999; 77(5): 1041-60.

56. Woods H, Proeve M. Relationships of mindfulness, self-compassion, and meditation experience with shameproneness. J Cogn Psychother. 2014; 28(1): 20-33.

57. Van Dam NT, Sheppard SC, Forsyth JP, Earleywine M. Self-compassion is a better predictor than mindfulness of symptom severity and quality of life in mixed anxiety and depression. J Anxiety Disord. 2011; 25(1): 123-30.

58. Mosewich AD, Kowalski KC, Sabiston CM, Sedgwick WA, Tracy JL. Self-Compassion: a potential resource for young women athletes. J Sport Exerc Psychol. 2011; 33(1): 103-23.

59. Barnard LK, Curry JF. The relationship of clergy burnout to self-compassion and other personality dimensions. Pastoral Psychol. 2011; 61: 149-63.

60. Lewis M. Self-conscious emotions. American Scientist. 1995; 83: 68-78.

61. Bergen-Cico D, Sanghyeon C. The mediating effects of mindfulness and self-compassion on trait anxiety. Mindfulness. 2014; 5: 505-19.

62. Hollis-Walker L, Colosimo K. Mindfulness, selfcompassion, and happiness in non-meditators: a theoretical and empirical examination. Pers Individ Dif. 2011; 50: 222-7.

63. Baer RA, Lykins ELB, Peters JR. Mindfulness and self-compassion as predictors of psychological well being in long-term meditators and matched nonmeditators. J Posit Psychol. 2012; 7(3): 230-8.

64. Charles Leland ST. Mindfulness, self-compassion, self-efficacy, and locus of control: examining relationships between four distinct but theoretically related concepts. Masters Thesis. New York. Pacific University. 2010.

65. Treynor W, Gonzalez R, Nolen-Hoeksema S. Rumination reconsidered: a psychometric analysis. Cognit Ther Res. 2003; 27(3): 247-59.

66. Verhaegen P, Joormann J, Khan R. Why we sing the blues: the relation between self-reflective rumination, mood, and creativity. Emotion. 2005; 5(2): 226-32.

67. Takano K, Tanno Y. Self-rumination, self-reflection, and depression: self-rumination counteracts the adaptive effect of self-reflection. Behav Res Ther. 2009; 47(3): 260-4.

68. Heydarinasab L, Ahmadvand Z, Shairi M. An investigation of the validity and reliability of psychometric characteristics of five facet mindfulness questionnaire in Iranian non-clinical samples. Journal of Behavioral Sciences. 2013; 7(3): 11-12.

69. Ghorbani N. Self narrated by self. Tehran:binesheno. 2013.

70. Raes F. Rumination and worry as mediators of the relationship between self-compassion and depression and anxiety. Personality and Individual Differences. 2010; 48(6): 757-61.

71. Ramel W, Goldin P R, Carmona PE, McQuaid JR. The effects of mindfulness meditation on cognitive processes and affect in patients with past depression. Cognit Ther Res. 2004; 28: 433-55.

72. Kabat-Zinn J. Full catastrophe living: using the wisdom of your body and. mind to face stress, pain and illness. New York: Dell Publishing. 1990.

73. Segal ZV, Teasdale JD, Williams JM. Mindfulness based cognitive therapy for depression. New York: The Guilford Press. 2002. 